\title{
Dynamics of Extreme Stratospheric Negative Heat Flux Events in an Idealized Model
}

\author{
ETIENNE DUNN-SigOUIN \\ Geophysical Institute, University of Bergen, and Bjerknes Centre for Climate Research, Bergen, Norway \\ TIFFANY SHAW \\ Department of the Geophysical Sciences, University of Chicago, Chicago, Illinois
}

(Manuscript received 5 September 2017, in final form 28 July 2018)

\begin{abstract}
Recent work has shown that extreme stratospheric wave-1 negative heat flux events couple with the troposphere via an anomalous wave- 1 signal. Here, a dry dynamical core model is used to investigate the dynamical mechanisms underlying the events. Ensemble spectral nudging experiments are used to isolate the role of specific dynamical components: 1) the wave-1 precursor, 2) the stratospheric zonal-mean flow, and 3 ) the higher-order wavenumbers. The negative events are partially reproduced when nudging the wave- 1 precursor and the zonal-mean flow whereas they are not reproduced when nudging either separately. Nudging the wave-1 precursor and the higher-order wavenumbers reproduces the events, including the evolution of the stratospheric zonal-mean flow. Mechanism denial experiments, whereby one component is fixed to the climatology and others are nudged to the event evolution, suggest higher-order wavenumbers play a role by modifying the zonal-mean flow and through stratospheric wave-wave interaction. Nudging all tropospheric wave precursors (wave-1 and higher-order wavenumbers) confirms they are the source of the stratospheric waves. Nudging all stratospheric waves reproduces the tropospheric wave-1 signal. Taken together, the experiments suggest the events are consistent with downward wave propagation from the stratosphere to the troposphere and highlight the key role of higher-order wavenumbers.
\end{abstract}

\section{Introduction}

The stratosphere couples with the tropospheric circulation across a range of time scales (Kidston et al. 2015). A manifestation of this coupling on intraseasonal time scales occurs during extreme phases of the northern annular mode in the Northern Hemisphere winter stratosphere, often referred to as vortex events (e.g., Baldwin and Dunkerton 2001; McLandress and Shepherd 2009; Gerber et al. 2010; Hitchcock et al. 2013). Weak vortex events, or stratospheric sudden warmings (SSWs), are followed by an equatorward shift of the zonal-mean tropospheric jet for several weeks (Baldwin and Dunkerton 2001; Limpasuvan et al. 2004). An opposite meridional jet shift occurs for strong vortex events (Baldwin and Dunkerton 2001; Limpasuvan et al. 2005). Downward coupling of the vortex is confirmed by studies showing a zonal-mean tropospheric response to a zonal-mean stratospheric perturbation (e.g., Polvani and Kushner 2002; Song and

Corresponding author: Etienne Dunn-Sigouin, etienne.dunn-sigouin @uib.no
Robinson 2004; Gerber and Polvani 2009; Hitchcock and Simpson 2014; Hitchcock and Haynes 2016; Smith and Scott 2016). Consistently, the accurate representation of extreme vortex events in forecast models enhances subseasonal to seasonal prediction skill of the wintertime extratropical troposphere (e.g., Sigmond et al. 2013; Tripathi et al. 2015; Scaife et al. 2016; Hansen et al. 2017).

An important driver of zonal-mean stratospheric variability is vertical coupling by planetary waves generated in the troposphere (Plumb 2010). Randel (1987) diagnosed vertical planetary wave coupling using crossspectral correlation analysis of daily wave-1 geopotential height. Upward wave coupling, defined as significant correlations when the troposphere leads the stratosphere, and downward wave coupling, defined as significant correlations when the stratosphere leads the troposphere, occur on the order of 5 days (Randel 1987; Perlwitz and Harnik 2003; Shaw et al. 2010). Another measure of the vertical coupling is the sign of the zonalmean meridional heat flux $\overline{v^{\prime} T^{\prime}}$, hereafter eddy heat flux, since it is proportional to the vertical wave group velocity according to linear theory. Here, primes denote 
eddies (deviations from the zonal mean) and the overbar denotes the zonal mean. Polvani and Waugh (2004) showed that weak vortex events are preceded by cumulative anomalous positive eddy heat flux whereas strong vortex events are preceded by cumulative anomalous negative eddy heat flux.

Recently, Dunn-Sigouin and Shaw (2015) and Shaw and Perlwitz (2013) used reanalysis data to show that extreme stratospheric negative eddy heat flux events, hereafter negative events, defined by the 5 th percentile of the total (anomaly plus climatology) 50-hPa wave- 1 heat flux in high latitudes $\left(60^{\circ}-90^{\circ} \mathrm{N}\right)$, involve a composite life cycle consistent with downward wave coupling. The evolution prior to negative events involves 1 ) an anomalous positive stratospheric wave- 1 heat flux precursor in high latitudes and 2) a zonal-mean bounded wave geometry in the stratosphere composed of a midlatitude meridional reflecting surface and a highlatitude vertical reflecting surface, which are defined by regions of negative meridional and vertical wavenumbers, respectively. The evolution during negative events involves 1) stratospheric Eliassen-Palm (EP) flux divergence and tropospheric convergence and 2) anomalous wave- 1 geopotential heights in the highlatitude troposphere associated with a poleward shift of the Atlantic jet. The life cycle is consistent with the prevailing view of downward wave coupling involving upward wave coupling (e.g., a wave-1 precursor) under specific zonal-mean-flow conditions (e.g., a bounded wave geometry), resulting in reflection (Harnik and Lindzen 2001; Perlwitz and Harnik 2003, 2004; Harnik 2009; Shaw et al. 2010, 2011; Lubis et al. 2016). This view of downward wave coupling does not include any role for higher-order wavenumbers (Palmer and Hsu 1983; Smith 1983; McIntyre 1982; Scinocca and Haynes 1998) or waves generated within the stratosphere (Mukougawa et al. 2017). The prevailing view of downward wave coupling is based on the diagnosis of reanalysis and model data; however, reanalysis and model data alone cannot confirm the underlying dynamical mechanisms of negative events, including the causal role of the stratosphere on the troposphere.

Dry dynamical core models are a useful tool for understanding the dynamical mechanisms of stratospheretroposphere coupling because they provide a simplified framework for isolating key physical processes. Here, we use ensemble spectral nudging following previous work (e.g., Douville 2009; Greatbatch et al. 2012) in a dry dynamical core to isolate the role of specific dynamical components of the life cycle. Nudging relaxes a component of the circulation toward a prescribed evolution to determine its impact on the free evolving circulation. The deterministic response is evaluated by averaging over an ensemble initialized from a set of independent initial conditions. We use nudging to quantify the role of 1) the wave-1 precursor, 2) the stratospheric zonal-mean flow, and 3) the higher-order wavenumbers in producing the negative event in the stratosphere and the tropospheric ingredients (high-latitude wave-1 EP-flux convergence and geopotential height anomalies). Our goal is to answer the following questions:

1) What is the role of the wave- 1 precursor and the stratospheric zonal-mean flow?

The prevailing view for downward wave coupling involves upward wave coupling (e.g., a wave-1 heat flux precursor) under specific zonal-mean-flow conditions (e.g., a bounded wave geometry), resulting in reflection (Harnik and Lindzen 2001; Perlwitz and Harnik 2003, 2004; Harnik 2009; Shaw et al. 2010, 2011; Lubis et al. 2016). Harnik (2009) suggested the wave-1 precursor creates the bounded wave geometry. If the prevailing view of downward wave coupling is the mechanism underlying negative events, then nudging the wave-1 precursor alone or in combination with the zonal-mean flow should reproduce the events. Conversely, nudging the zonal-mean flow alone should not reproduce the events.

2) What is the role of higher-order planetary wavenumbers?

Higher-order wavenumbers could play a role during negative events by generating the wave- 1 precursor (Scinocca and Haynes 1998). Higher-order wavenumbers could also play a role by modifying the stratospheric zonal-mean flow. McIntyre (1982) proposed that higher-order wavenumbers can produce enhanced positive heat fluxes by sharpening the vortex edge. Higher-order wavenumbers could play a similar role during negative events by shaping the stratospheric wave geometry. Finally, higher-order wavenumbers could produce negative events through stratospheric wave-wave interaction via two mechanisms. The first occurs through stratospheric wave breaking of tropospherically forced waves, where enstrophy is transferred from larger to smaller scales. Palmer and Hsu (1983) proposed that stratospheric wave-1 breaking produces higher-order wavenumbers that drive sudden stratospheric cooling/acceleration events, which occur when the stratospheric eddy heat flux is negative (Shaw and Perlwitz 2014; Lubis et al. 2017). The second mechanism occurs when a given tropospheric zonal wavenumber propagates upward relative to a distorted polar vortex and projects onto higher or lower wavenumbers defined on a latitude circle (Smith 1983). Nudging the higher-order wavenumbers alone or in combination with the other dynamical components should 
demonstrate if and how they play a role during negative events.

3) What is the role of upward wave coupling?

During negative events, downward wave coupling could result from 1) upward wave coupling involving a tropospheric wave source consistent with the prevailing view in the literature or 2) a stratospheric wave source. If the upward wave coupling plays a role during negative events, then nudging the tropospheric wave precursors should reproduce the stratospheric wave precursors and the stratospheric and tropospheric evolution during the events.

4) Does the stratosphere play a causal role?

During negative events, the reversal of the stratospheric heat flux and tropospheric wave-1 signal occurs almost simultaneously (Figs. $2 \mathrm{~b}$ and $5 \mathrm{~d}$ in Dunn-Sigouin and Shaw 2015). Furthermore, negative eddy heat fluxes do not necessarily imply downward wave coupling if the conditions are not linear (Plumb 2010). If the stratosphere plays a causal role during negative events, then nudging the stratospheric wave evolution should reproduce the tropospheric ingredients during negative events.

Section 2 presents the idealized model, reanalysis data, diagnostics, and experiments used in this study. Section 3 presents the model results. Specifically, we compare the composite life cycle of negative events in the model and reanalysis in section 3a. We quantify the role of the dynamical components in reproducing negative events in section $3 \mathrm{~b}$. We test the role of the higher-order wavenumbers in section $3 \mathrm{c}$. We quantify the role of upward wave coupling in section $3 \mathrm{~d}$. We test the causal role of the stratosphere on the troposphere in section 3e. Conclusions are summarized and discussed in section 4 .

\section{Idealized model, reanalysis data, diagnostics, and experiments}

\section{a. Dry dynamical core}

We make use of the Geophysical Fluid Dynamics Laboratory's spectral dry dynamical core model. The model integrates the primitive equations on a sphere and is driven by idealized physics. The equations are forced with Newtonian relaxation toward a specified zonally symmetric equilibrium temperature profile and Rayleigh drag near the surface and in the stratosphere near the model lid. The analytic expressions of the thermal and momentum forcings are provided in Held and Suarez (1994) and Polvani and Kushner (2002) and represent perpetual winter conditions. The model horizontal resolution is triangular truncation 42 (T42) and the vertical resolution is 40 hybrid-sigma vertical levels. Stationary waves are generated in the model via Gaussian zonal wave- 1 topography spanning $25^{\circ}-65^{\circ} \mathrm{N}$ centered at $45^{\circ} \mathrm{N}$, following Eq. (1) in Gerber and Polvani (2009).

Gerber and Polvani (2009) used a dry dynamical core model with wave-2 topography to capture the key ingredients of weak vortex events (integration 9 in their Table 1). Here, we modify their model parameters to generate a control (CTL) simulation with wave-1 topography that qualitatively captures the key ingredients of negative events outlined in the introduction (Table 1). The CTL simulation is integrated for 20000 days where the first 500 days of model spinup are discarded. All data are linearly interpolated to pressure coordinates and daily data are used for all calculations including fluxes. Meridional averages are cosine (mass) weighted and a 5-day running-mean smoothing is applied when plotting. Anomalies are defined relative to the model time-mean climatology.

\section{b. Reanalysis data}

We compare the CTL simulation to daily ERA-Interim data (Dee et al. 2011) during Northern Hemisphere (NH) January-March (JFM) from 1979 to 2012, which represents the period of maximum stratosphere-troposphere planetary wave coupling in the Northern Hemisphere (Shaw et al. 2010). Anomalies are defined relative to the daily mean seasonal cycle.

\section{c. Diagnostics}

\section{1) EXTREME STRATOSPHERIC NEGATIVE WAVE-1 HEAT FLUX EVENTS}

Negative events in the reanalysis and the CTL simulation are defined by extreme values of the daily total (anomaly plus climatology) zonal-mean wave-1 meridional heat flux $\left(\overline{v^{\prime} T^{\prime}}{ }_{k=1}\right)$ averaged from $60^{\circ}$ to $90^{\circ} \mathrm{N}$ at $50 \mathrm{hPa}$, as in Dunn-Sigouin and Shaw (2015). The highlatitude heat flux is used because stratospheric planetary wave-1 variability peaks in high latitudes in reanalysis data (Shaw and Perlwitz 2013). Negative events are identified when the 5-day running-mean high-latitude heat flux drops below the 5th percentile of the climatological distribution. The central date (day 0 ) of the events is defined at the day of minimum or maximum high-latitude heat flux and each event must be separated by a minimum of 15 days.

We note that a subset of events in the CTL simulation are preceded by a reversal of the stratospheric zonalmean zonal wind, making it impossible to test the role of the zonal-mean flow associated with a bounded wave geometry, which is a key feature of the composite reanalysis event and the prevailing view of downward 
TABLE 1. Length of integration and model parameters for the CTL simulation. The CTL simulation is generated by modifying key parameters from integration 9 in Table 1 in Gerber and Polvani (2009), where all other parameters are equal including the surface topography. The Gerber and Polvani (2009) model parameters are included for comparison. The parameters control the surface topography and latitude-pressure structure of the zonal-mean thermal relaxation temperature $T_{\text {eq }}$. Specifically, $h_{o}$ and $k$ are the height and zonal wavenumber of the topography, respectively; $\varepsilon$ controls the hemispheric asymmetry of the meridional gradient of $T_{\mathrm{eq}}$ in the troposphere; $p_{t}$ is the pressure level where $T_{\text {eq }}$ transitions from the troposphere to the stratosphere; $p_{\text {sp }}$ is the lowest pressure level of the stratospheric sponge layer; $\gamma$ controls the stratospheric high-latitude lapse rate of $T_{\text {eq }}$, which transitions smoothly from the U.S. standard temperature profile at the equator; and $\delta \phi$ controls the meridional gradient of the transition while $\phi_{o}$ controls the central latitude of the gradient of the transition. See Polvani and Kushner (2002) and Gerber and Polvani (2009) for the exact equations defining $T_{\text {eq }}$ and the surface topography.

\begin{tabular}{lccccccccc}
\hline \hline & Length (days) & $h_{o}(\mathrm{~m})$ & $k$ & $\varepsilon\left(^{\circ}\right)$ & $p_{t}(\mathrm{hPa})$ & $p_{\text {sp }}(\mathrm{hPa})$ & $\gamma\left(\mathrm{K} \mathrm{km}^{-1}\right)$ & $\delta \phi\left(^{\circ}\right)$ & $\phi_{o}\left({ }^{\circ}\right)$ \\
\hline CTL & 20000 & 4000 & 1 & 20 & 200 & 1 & -6.5 & 20 & 60 \\
Gerber and Polvani (2009) & - & 3000 & 2 & 10 & 100 & 0.5 & -4 & 10 & 50 \\
\hline
\end{tabular}

wave coupling. Therefore, in the model and reanalysis, we discard negative events where the zonal-mean zonal wind at $10 \mathrm{hPa}$ averaged over $60^{\circ}-90^{\circ} \mathrm{N}$ is negative anytime within 10 days prior to the events. The additional criteria remove negative events that occur following SSWs (Kodera et al. 2016; Mukougawa et al. 2017), which represent $30 \%$ of events in the CTL simulation and reanalysis. Finally, as a result of computational and storage constraints, we choose a subset of the strongest 15 events in the CTL simulation (corresponding to the 0.7 th percentile, $-21 \mathrm{~K} \mathrm{~m} \mathrm{~s}^{-1}$ ) for comparison with the 20 events from the reanalysis (5th percentile, $\left.-13.5 \mathrm{~K} \mathrm{~m} \mathrm{~s}^{-1}\right)$. The key results of this study are reproduced using the strongest 50 events (2nd percentile, $-12.8 \mathrm{~K} \mathrm{~m} \mathrm{~s}^{-1}$ ), which have similar composite life cycles.

\section{2) WAVE GEOMETRY}

We analyze the structure of the stratospheric zonalmean flow during negative events using the wave geometry diagnostic outlined in Harnik and Lindzen (2001). The wave geometry diagnostic partitions the traditional index of refraction $n^{2}$ into vertical $\mathrm{m}^{2}$ and meridional $l^{2}$ wavenumber components. The wavenumbers are diagnosed from the solution of the linearized quasigeostrophic PV equation for a given zonal-mean zonal wind and temperature and stationary zonal-wavenumber $k=1$ surface boundary conditions. Waves propagate in the vertical (meridional) direction when $m^{2}>0\left(l^{2}>0\right)$ and are evanescent when $m^{2}<0\left(l^{2}<0\right)$. In practice, $l^{2}<0$ occurs when the $\mathrm{PV}$ gradient is weak or negative because of the negative meridional curvature of the zonal-mean zonal wind while $m^{2}<0$ occurs when the $\mathrm{PV}$ gradient is weak or negative as a result of the negative vertical shear of the zonal-mean zonal wind (Harnik and Lindzen 2001; Perlwitz and Harnik 2003). We use the wave geometry diagnostic to calculate vertical reflecting surfaces $\left(m^{2}=0\right)$ and meridional reflecting surfaces $\left(l^{2}=0\right)$ in the model and reanalysis following previous work (Harnik and Lindzen 2001; Perlwitz and
Harnik 2003; Shaw et al. 2010, 2011; Shaw and Perlwitz 2013; Lubis et al. 2016). The wave geometry is calculated from daily fields and then averaged.

\section{d. Nudging experiments}

The role of the specific dynamical components during negative events is evaluated using ensemble spectral nudging experiments following previous work. Nudging forces the spectral tendencies of model vorticity, divergence, temperature, and surface pressure via Newtonian relaxation toward a prescribed reference state, for example, $-C(p)\left(X-X_{o}\right) / \tau$, where $X$ is the instantaneous value of a given field, $X_{o}$ is the referencestate field, $\tau$ is the nudging time scale, and $C(p)$ is the pressure-dependent nudging coefficient that controls the vertical structure of nudging. The wave- 1 precursor, stratospheric zonal-mean flow, and higher-order wavenumbers are controlled by nudging their corresponding wavenumbers toward the CTL event evolution. The spectral nudging and wavenumber decomposition for the reference states are carried out on the native hybridsigma vertical grid. Higher-order wavenumbers are defined to be wavenumbers $k \geq 2$; however, the key results are similar using wavenumbers $k 2-4$ (experiments 17-20 in Table 2; see Fig. A2). A nudging time scale $\tau$ equal to $1 / 10$ day is used. The response is determined by averaging over an ensemble initialized from a set of independent initial conditions taken from the CTL simulation every 50 days. Each ensemble is composed of 50 members and each member is integrated for 51 days, representing lags -30 to +20 centered around each negative event. The experiments are summarized in Table 2.

To isolate the importance of the timing of the dynamical components, the nudging is varied with time. For example, the wave- 1 precursor is nudged before the event from day -20 to day -8 to capture the anomalous positive wave-1 heat flux prior to the event without nudging the reversal of the stratospheric heat flux or the tropospheric ingredients. We note qualitatively similar 
TABLE 2. Summary of the ensemble spectral nudging experiments as a function of zonal wavenumber, vertical domain, and lag relative to each event from the CTL simulation. The stratospheric and tropospheric vertical domains are defined as hybrid-sigma levels above and below $137 \mathrm{hPa}$, respectively. An ensemble is produced for each of the 15 CTL events. Ensembles are composed of 50 members and each member is integrated for 51 days, representing lags from -30 to +20 centered on each event.

\begin{tabular}{|c|c|c|c|}
\hline Expt & Wavenumber & Vertical domain & Lag (days) \\
\hline 1 & $k 1$ & All & -20 to -8 \\
\hline 2 & $k 0$ & All & -20 to +20 \\
\hline \multirow[t]{2}{*}{3} & $k 0$ & All & -20 to +20 \\
\hline & $k 1$ & All & -20 to -8 \\
\hline 4 & $k \geq 2$ & All & -20 to -20 \\
\hline \multirow[t]{2}{*}{5} & $k 0$ & All & -20 to +20 \\
\hline & $k \geq 2$ & All & -20 to +20 \\
\hline \multirow[t]{2}{*}{6} & $k 1$ & All & -20 to -8 \\
\hline & $k \geq 2$ & All & -20 to +20 \\
\hline 7 & $k \geq 2$ (random) & All & -20 to +20 \\
\hline \multirow[t]{2}{*}{8} & $k 1$ (climatology) & All & -20 to -8 \\
\hline & $k \geq 2$ & All & -20 to +20 \\
\hline \multirow[t]{2}{*}{9} & $k 0$ (climatology) & All & -20 to +20 \\
\hline & $k \geq 2$ & All & -20 to +20 \\
\hline \multirow[t]{3}{*}{10} & $k 0$ (climatology) & All & -20 to +20 \\
\hline & $k 1$ (climatology) & All & -20 to -8 \\
\hline & $k \geq 2$ & & -20 to +20 \\
\hline 11 & $k 1$ & Troposphere & -20 to -8 \\
\hline \multirow[t]{2}{*}{12} & $k 1$ & Troposphere & -20 to -8 \\
\hline & $k \geq 2$ & Troposphere & -20 to -5 \\
\hline \multirow[t]{2}{*}{13} & $k 1$ (random) & Troposphere & -20 to -8 \\
\hline & $k \geq 2$ (random) & Troposphere & -20 to -5 \\
\hline 14 & $k \geq 1$ & Stratosphere & -20 to +20 \\
\hline 15 & $k \geq 1$ & Stratosphere & -5 to +5 \\
\hline 16 & $k 1$ & Stratosphere & -20 to +20 \\
\hline \multirow[t]{2}{*}{17} & $k 1$ & All & -20 to -8 \\
\hline & $k 2-4$ & All & -20 to +20 \\
\hline \multirow[t]{3}{*}{18} & $k 0$ (climatology) & All & -20 to +20 \\
\hline & $k 1$ (climatology) & All & -20 to -8 \\
\hline & $k 2-4$ & All & -20 to +20 \\
\hline \multirow[t]{2}{*}{19} & $k 1$ & Troposphere & -20 to -8 \\
\hline & $k 2-4$ & Troposphere & -20 to -5 \\
\hline 20 & $k 1-4$ & Stratosphere & -20 to +20 \\
\hline
\end{tabular}

results are found when nudging the wave- 1 precursor from day -20 to -10 or from day -20 to -5 .

To isolate the role of stratospheric versus tropospheric dynamics, the nudging is varied with height. Specifically, stratospheric nudging varies linearly from 1 above $p_{t}$ to 0 below $p_{b}$ :

$$
C(p)=\left\{\begin{array}{ccc}
1, & \text { if } & p<p_{t} \\
\frac{p-p_{b}}{p_{t}-p_{b}}, & \text { if } & p_{t}<p<p_{b} . \\
0, & \text { if } & p>p_{b}
\end{array}\right.
$$

Similarly, tropospheric nudging varies linearly from 0 above $p_{t}$ to 1 below $p_{b}$ :

$$
C(p)=\left\{\begin{array}{ccc}
0, & \text { if } & p<p_{t} \\
\frac{p-p_{t}}{p_{b}-p_{t},} & \text { if } & p_{t}<p<p_{b} . \\
1, & \text { if } & p>p_{b}
\end{array}\right.
$$

On hybrid-sigma levels, the model-integrated tropopause varies from 137 to $298 \mathrm{hPa}$ from the tropics to the Arctic. The pressure values corresponding to the tropopause are defined in hybrid-sigma coordinates as equivalent pressure levels assuming a constant surface pressure of $1000 \mathrm{hPa}$. Here, we apply stratospheric or tropospheric nudging above or below $137 \mathrm{hPa}$; however, similar results are obtained if $298 \mathrm{hPa}$ is used. Thus, stratospheric nudging has values of $p_{t}=80 \mathrm{hPa}$ and $p_{b}=137 \mathrm{hPa}$ and tropospheric nudging has values of $p_{t}=137 \mathrm{hPa}$ and $p_{b}=221 \mathrm{hPa}$. The coefficient multiplying the surface pressure nudging tendencies are set to 1 and 0 for tropospheric and stratospheric nudging, respectively.

We note that since the model is integrated on hybridsigma levels, nudging a given wavenumber in the sigmacoordinate troposphere (e.g., $k \geq 2$ ) could inadvertently influence other wavenumbers (e.g., $k 1$ ) in the pressure coordinate troposphere and stratosphere. All tropospheric wave-1 responses are very similar when plotted in hybrid-sigma versus pressure coordinates, showing they are not primarily driven by issues with the nudging because the nudging explicitly excludes wave 1 in hybrid-sigma coordinates by construction. Furthermore, nudging the higher-order wavenumbers toward random events or toward the CTL simulation for 2000 days (section 3b) does not systematically reproduce the stratospheric wave-1 heat flux, suggesting issues with the nudging methodology are not the primary cause of the stratospheric wave-1 precursor.

Each experiment performs identical nudging for all 15 negative events from the CTL simulation. The results are shown as the composite ensemble mean for comparison with the composite CTL event. Anomalies are defined relative to the CTL climatology for all days. The statistical significance of each experiment is quantified using the spread of ensemble means and reported at the 95\% level using a two-tailed Student's $t$ test.

\section{Results}

We begin by comparing the dynamical components and ingredients of the composite negative event in reanalysis and the CTL simulation. Next, we quantify the role of the dynamical components in producing the ingredients of negative events in the CTL simulation by nudging each component separately and in combination. 
(a) reanalysis

VT k1 anomaly 60-90N

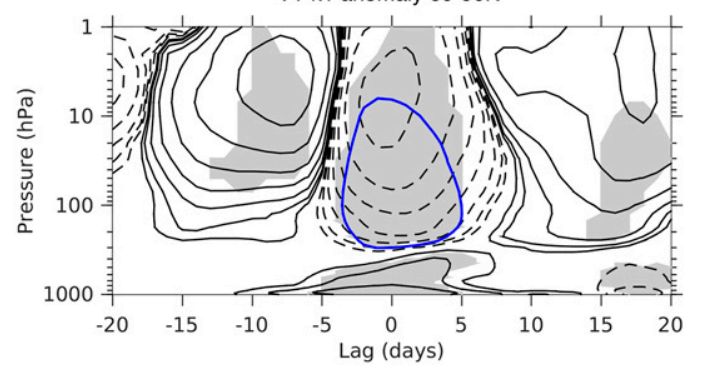

(c) reanalysis

$\mathrm{U}, \mathrm{m}^{\wedge} 2$ \& $\mathrm{I}^{\wedge} 2$ day -5 to -1

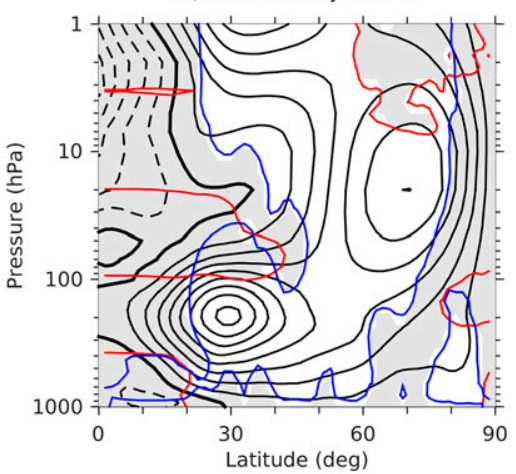

(e) reanalysis

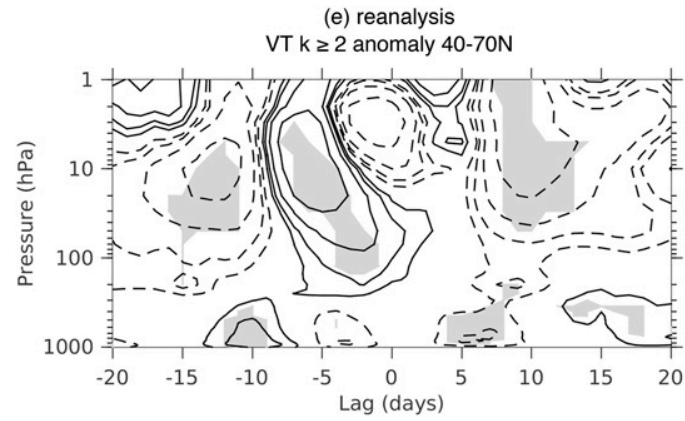

(b) CTL

VT k1 anomaly 60-90N

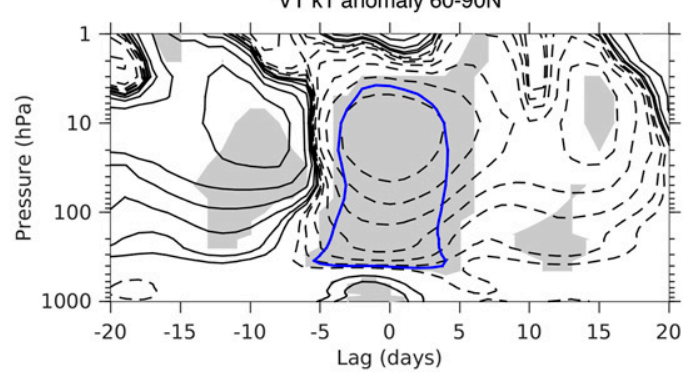

(d) CTL

$U, m^{\wedge} 2$ \& $I^{\wedge} 2$ day -5 to -1

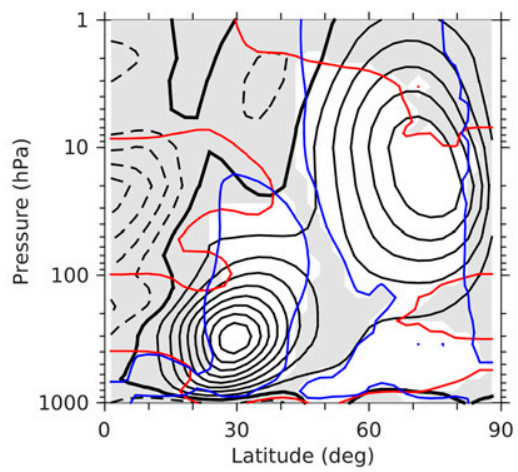

(f) CTL

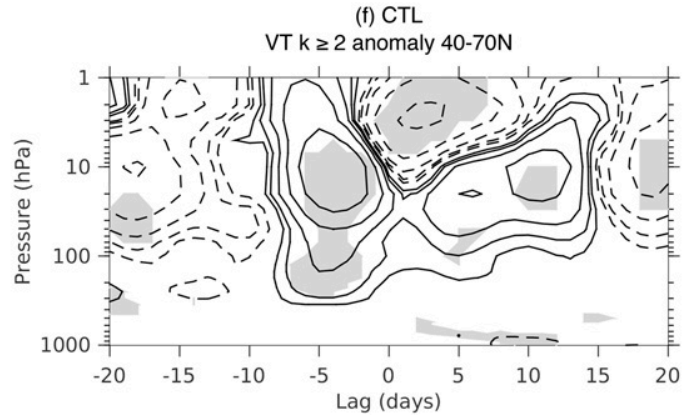

FIG. 1. Dynamical components of the composite negative events in the (a),(c),(e) reanalysis and (b),(d),(f) CTL simulation. (top) Wave-1 precursor, shown as the evolution of the anomalous high-latitude wave-1 meridional heat flux. The black contours are $\pm[1,2,4,8,16,32, \ldots] \mathrm{K} \mathrm{m} \mathrm{s}^{-1}$ and dashed contours are negative. The blue contour denotes total negative (anomaly plus climatology) values. Shading indicates statistical significance at the $95 \%$ level based on a two-tailed $t$ test. (middle) Zonal-mean flow, shown as the zonal-mean zonal wind (black contours) and zero contour of the squared vertical and meridional wavenumber (red and blue contours, respectively) averaged over days -5 to -1 . The thin black contour interval is $5 \mathrm{~m} \mathrm{~s}^{-1}$ and the thick black contour denotes the zero line. Shading denotes regions where either the squared vertical or meridional wavenumber is negative. (bottom) Higherorder wavenumbers, as in the top panels, but for $k \geq 2$ meridional heat flux averaged over $40^{\circ}-70^{\circ} \mathrm{N}$. Shading indicates statistical significance at the $95 \%$ level based on a two-tailed $t$ test.

\section{a. Comparing events in the model and reanalysis}

In terms of the stratospheric components and ingredients, the composite negative event in reanalysis exhibits a high-latitude wave-1 precursor involving anomalous positive stratospheric heat fluxes from day -13 to day -7 , followed by the negative event from day -4 to day +4 (Fig. 1a, blue contour). The CTL simulation qualitatively captures the evolution prior to and during the event but not after (Fig. 1b). The zonalmean flow prior to the reanalysis event involves a poleward-shifted stratospheric vortex forming a bounded wave geometry, with a meridional reflecting surface in midlatitudes (Fig. 1c, blue contours) and a vertical reflecting surface in high latitudes (Fig. 1c, red contours), which is qualitatively captured by the CTL simulation (Fig. 1d). Finally, higher-order wavenumbers during the reanalysis event involve an anomalous 
(a) reanalysis

EPFD k1 anomaly 60-90N

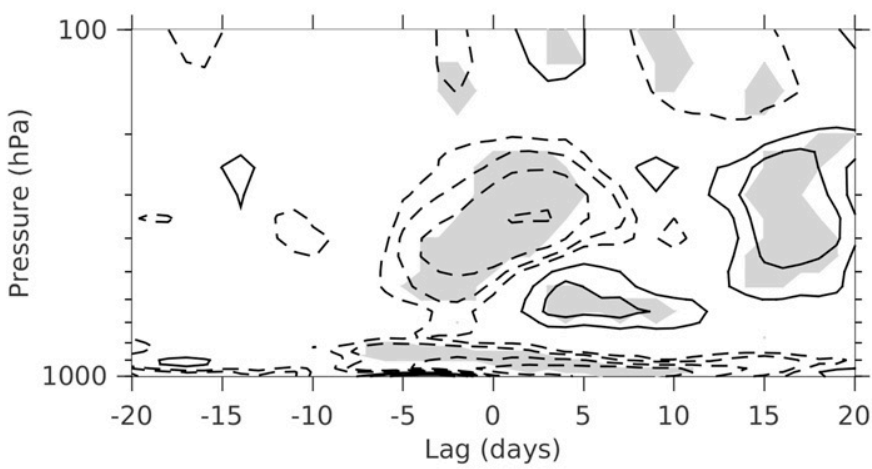

(c) CTL

EPFD k1 anomaly 60-90N

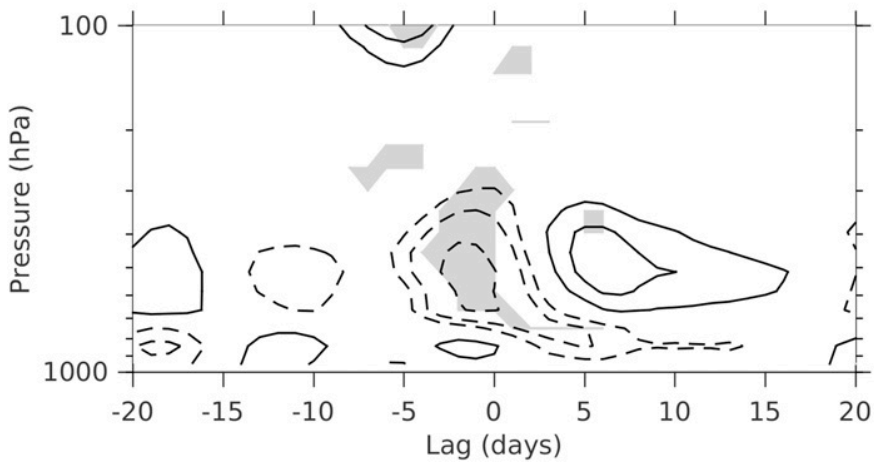

(b) reanalysis

Z k1 anomaly $400 \mathrm{hPa}$ day -3 to +3



(d) CTL

Z k1 anomaly $400 \mathrm{hPa}$ day -3 to +3

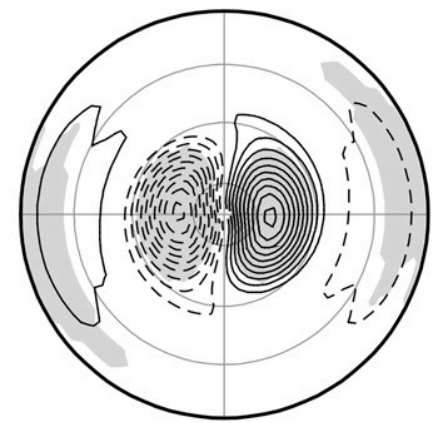

FIG. 2. Tropospheric ingredients of the composite negative events in (a),(b) reanalysis and (c),(d) the CTL simulation. (left) Evolution of the anomalous high-latitude wave-1 EP-flux divergence. The EP fluxes are computed for the primitive equations in log-pressure coordinates (Andrews et al. 1987) and then 5-day smoothed. The black contours are $\pm[0.5,1,2,4,8,16, \ldots] \mathrm{m} \mathrm{s}^{-1}$ day $^{-1}$ and dashed contours are negative. (right) Anomalous wave- 1 geopotential height at $400 \mathrm{hPa}$ averaged over days -3 to +3 . Interval for the black contours is $10 \mathrm{~m}$. Shading indicates statistical significance at the $95 \%$ level based on a two-tailed $t$ test.

positive stratospheric heat flux precursor from day -7 to day -2 in midlatitudes $\left(40^{\circ}-70^{\circ} \mathrm{N}\right.$; Fig. 1e). Once again, the CTL simulation qualitatively captures the higherorder wavenumbers prior to the event but not after (Fig. 1f). The higher-order wavenumber heat flux precursor averaged from $40^{\circ}$ to $70^{\circ} \mathrm{N}$ at $10 \mathrm{hPa}$ corresponds to the 90th percentile in reanalysis and the CTL simulation, showing they exhibit an extreme positive event.

In terms of tropospheric ingredients, the composite negative event in the reanalysis involves tropospheric EP-flux convergence on days -3 to +3 (Fig. 2a), which is dominated by the vertical component (not shown). During this time, the reanalysis event exhibits a largeamplitude tropospheric wave-1 geopotential height anomaly at high latitudes (Fig. 2b), which dominates the total geopotential height anomaly (Fig. 5b in DunnSigouin and Shaw 2015). The high-latitude EP-flux divergence and geopotential height responses are qualitatively captured by the CTL simulation (Figs. 2c,d).
However, the CTL simulation does not capture the poleward shift of the Atlantic jet during the reanalysis event (Fig. 5d in Dunn-Sigouin and Shaw 2015). This is not surprising because the CTL simulation does not capture the tilt of the Atlantic storm track seen in reanalysis (not shown).

Overall, the CTL simulation qualitatively captures the dynamical components and ingredients of negative events in reanalysis before and during the events but not after. In particular, the CTL simulation captures the two requirements involved in the prevailing view of downward wave coupling: the wave- 1 precursor and the zonal-mean bounded wave geometry. The results also suggest a role for higher-order wavenumbers because they are extreme in the stratosphere prior to negative events in reanalysis and the CTL simulation. Given the model performance, in the following subsection we focus on understanding the dynamics before and during negative events. 
(a) k1, experiment 1

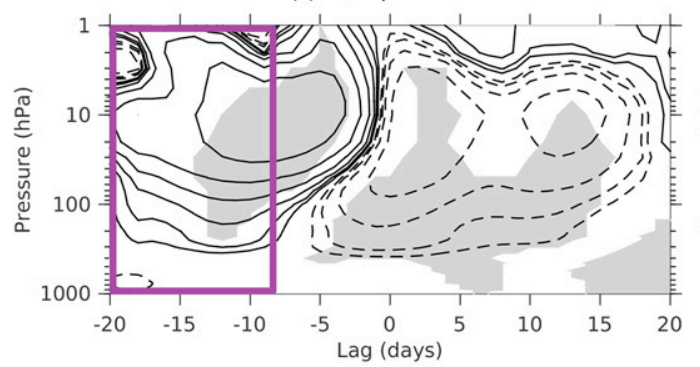

(c) $\mathrm{k} 0+\mathrm{k} 1$, experiment 3

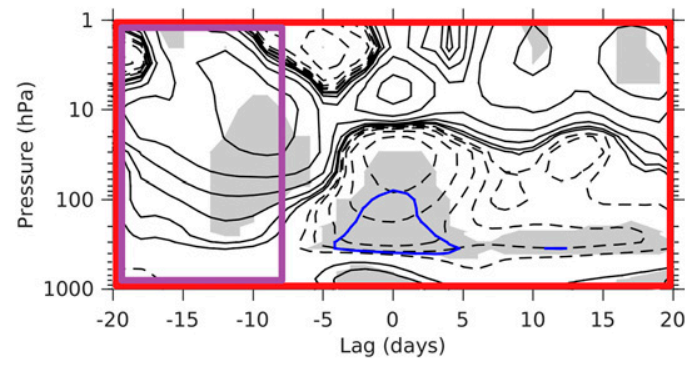

(e) $\mathrm{kO}+\mathrm{k} \geq 2$, experiment 5

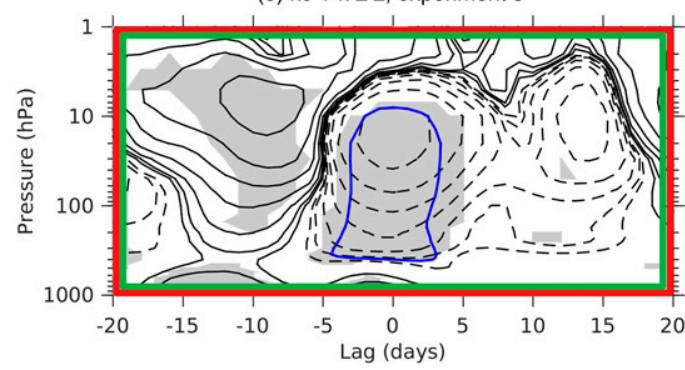

(b) k0, experiment 2

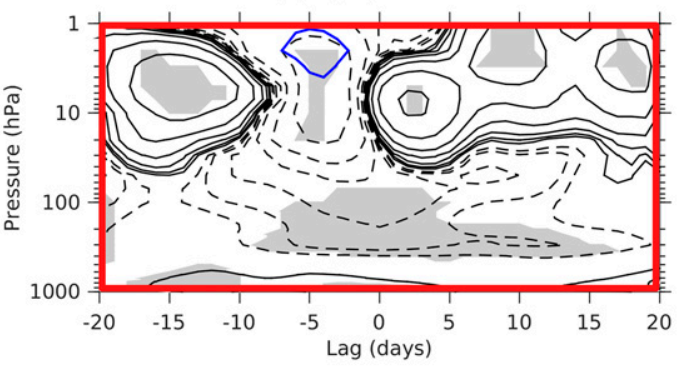

(d) $\mathrm{k} \geq 2$, experiment 4

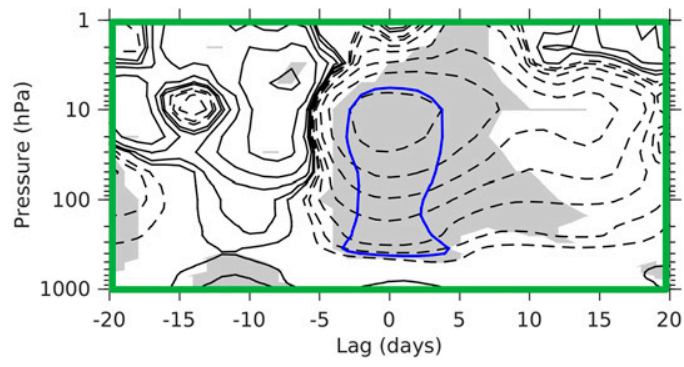

(f) $\mathrm{k} 1+\mathrm{k} \geq 2$, experiment 6

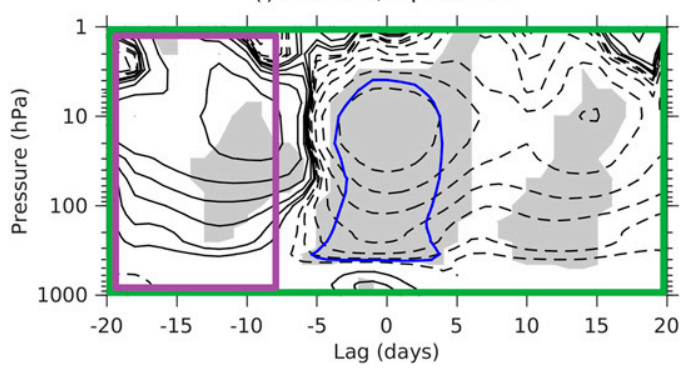

FIG. 3. As in Figs. 1a and 1b, but for composite ensemble-mean responses for experiments (a) 1 to (f) 6 . The red, purple, and green boxes denote the lag-pressure domains where the zonal-mean flow and wavenumbers $k 1$ and $k \geq 2$ are nudged, respectively.

\section{b. Quantifying the role of the dynamical components}

In this section, we quantify the role of the dynamical components in producing negative events in the CTL simulation. We begin by testing the prevailing view of downward wave coupling by nudging the wave- 1 precursor and the zonal-mean flow separately (experiments 1 and 2) and in combination (experiment 3 ). If the prevailing view of downward wave coupling in the literature is correct, then nudging the wave- 1 precursor alone or in combination with the zonal-mean flow should be sufficient to reproduce negative events while nudging the zonalmean flow alone should not reproduce the events.

Nudging the wave- 1 precursor produces anomalous negative heat fluxes in the stratosphere but does not lead to a heat flux reversal (Fig. 3a). Specifically, the wave-1 precursor reproduces $19 \%$ of the anomalous high-latitude wave- 1 heat flux at $50 \mathrm{hPa}$, the level where negative events are defined, averaged over days -3 to +3 during the composite CTL event (Fig. 4a). Nudging the zonal-mean flow alone does not produce either a wave-1 precursor or a heat flux reversal (Fig. 3b), reproducing $15 \%$ of the anomalous heat flux at $50 \mathrm{hPa}$ (Fig. $4 \mathrm{~b}$ ). Finally, nudging the zonal-mean flow and the wave- 1 precursor leads to a heat flux reversal in the lower stratosphere (Fig. 3c), reproducing $37 \%$ of the anomalous heat flux at $50 \mathrm{hPa}$ (Fig. 4c) but $59 \%$ at $100 \mathrm{hPa}$.

Thus far, the results provide support for the prevailing view of downward wave coupling since the wave-1 precursor and the zonal-mean flow in combination partially reproduce negative events in the lower stratosphere $(59 \%)$ while the zonal-mean flow alone does not. However, nudging the wave-1 precursor alone does not reproduce negative events in contrast to the hypothesis of Harnik (2009). In addition, the wave-1 precursor and the zonal-mean flow capture only $37 \%$ of negative events in the midstratosphere, suggesting a missing component. Therefore, we next quantify the role of the higher-order wavenumbers by nudging the higher-order wavenumbers alone (experiment 4) and in 
(a) k1, experiment 1 (19\%)

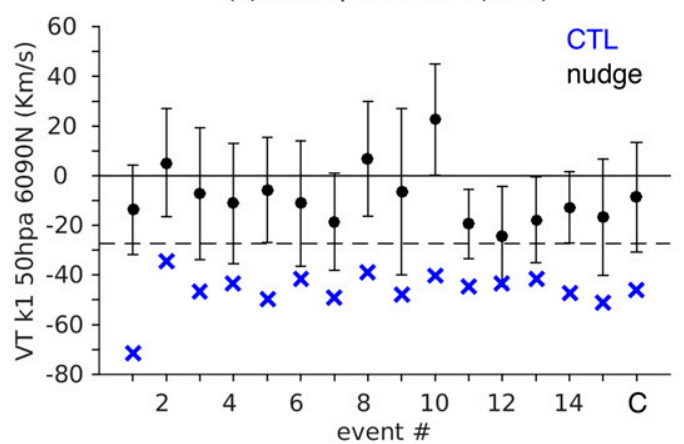

(c) $\mathrm{k} 0+\mathrm{k} 1$, experiment $3(37 \%)$

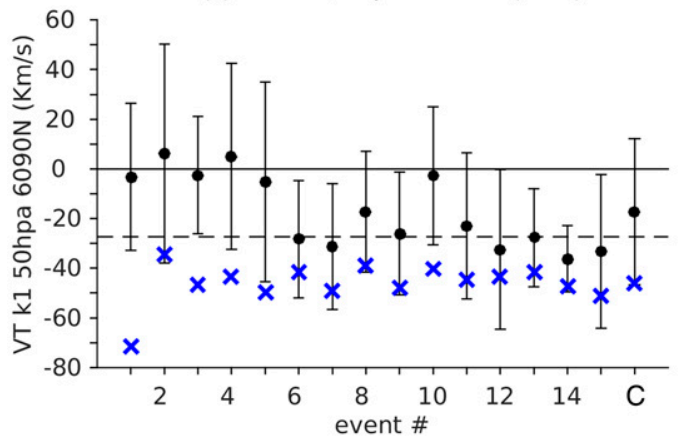

(e) $k 0+k \geq 2$, expeirment $5(87 \%)$

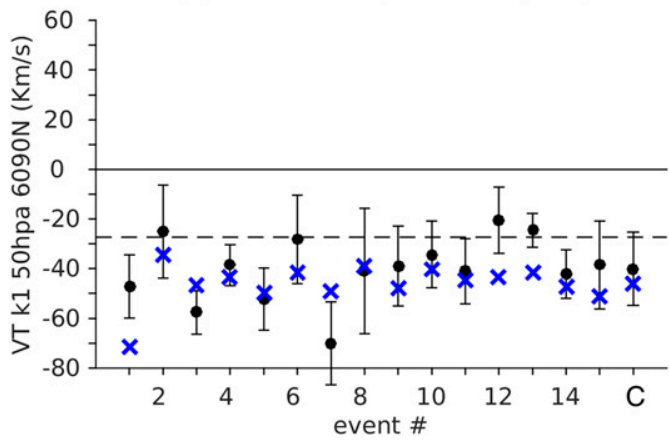

(b) k0, experiment $2(15 \%)$



(d) $k \geq 2$, experiment $4(70 \%)$

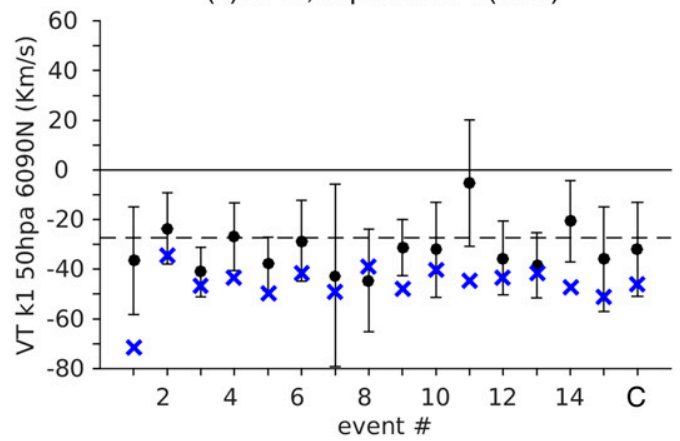

(f) $\mathrm{k} 1+\mathrm{k} \geq 2$, experiment $6(96 \%)$

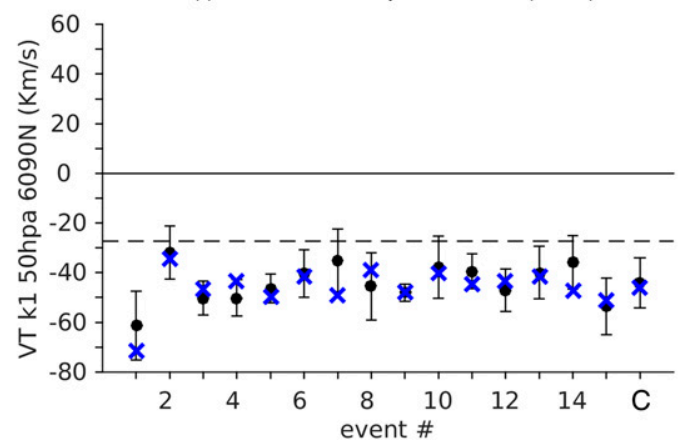

FIG. 4. Anomalous high-latitude wave- 1 heat flux at $50 \mathrm{hPa}$ averaged over days -3 to +3 during ensembles for individual events in experiments (a) 1 to (f) 6 . Blue $\times$ symbols indicate the CTL event values, black dots indicate the ensemble-mean values, and error bars denote the one-standard-deviation ensemble ranges. The composite CTL and ensemble-mean event is shown as the right-most value. The horizontal dashed line denotes total (anomaly plus climatology) negative values. The percentage value for each panel title denotes the percentage of the composite CTL event anomaly reproduced by the composite ensemble-mean anomaly.

combination with the zonal-mean flow (experiment 5) and the wave-1 precursor (experiment 6).

Nudging the higher-order wavenumbers leads to a heat flux reversal throughout the stratosphere (Fig. 3d), reproducing $70 \%$ of the anomalous heat flux at $50 \mathrm{hPa}$ during the composite CTL event (Fig. 4d). The composite event response shows a hint of the wave- 1 precursor although it is not statistically significant. Additional analysis shows the wave- 1 precursor is partially reproduced during several individual events when nudging the higher-order wavenumbers (see Fig. A1a in the appendix). Nudging the zonal-mean flow and the higher-order wavenumbers leads to a wave- 1 precursor and a heat flux reversal (Fig. 3e), reproducing $87 \%$ of the anomalous heat flux at $50 \mathrm{hPa}$ (Fig. 4e). Finally, nudging the wave- 1 precursor and the higher-order wavenumbers also leads to a heat flux reversal (Fig. 3f), reproducing $96 \%$ of the anomalous heat flux at $50 \mathrm{hPa}$ (Fig. 4f). A similar result is found when nudging the wave- 1 precursor and wavenumbers $k 2-4$ (Fig. A2a). 
(a) $\mathrm{k} 1$, experiment 1 VT $k \geq 2$ anomaly $40-70 \mathrm{~N}$

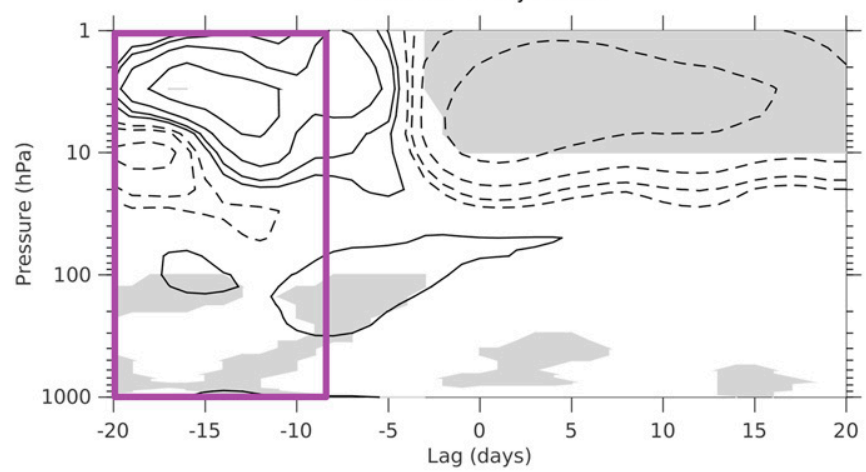

(c) $\mathrm{k} 0$, experiment 2

VT $k \geq 2$ anomaly $40-70 \mathrm{~N}$

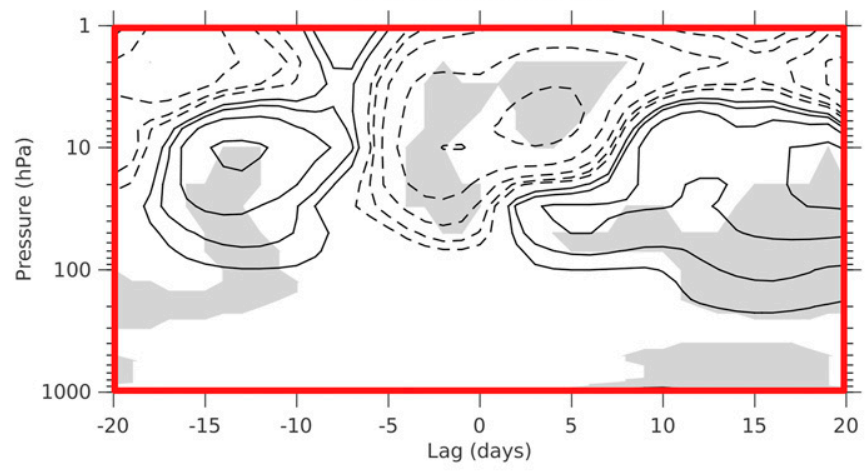

(e) $\mathrm{k} 0+\mathrm{k} 1$, experiment 3

VT $k \geq 2$ anomaly $40-70 \mathrm{~N}$

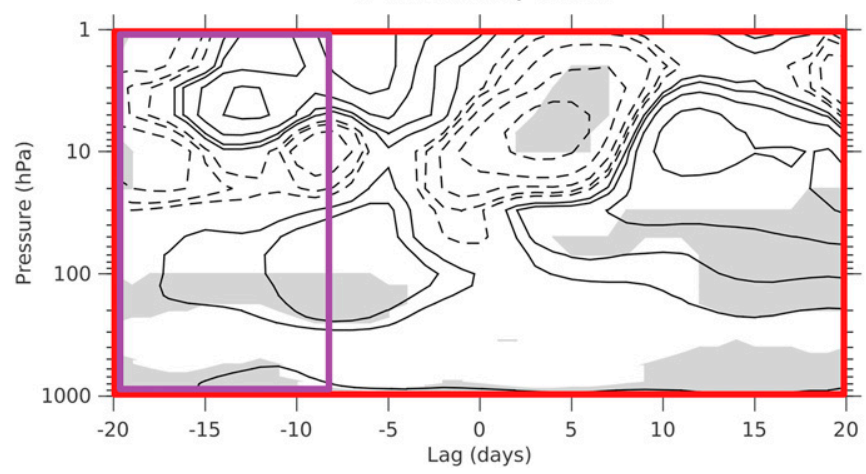

(b) $\mathrm{k} 1$, experiment 1

$\mathrm{U}, \mathrm{m}^{\wedge} 2$ \& $1^{\wedge} 2$ day -5 to -1

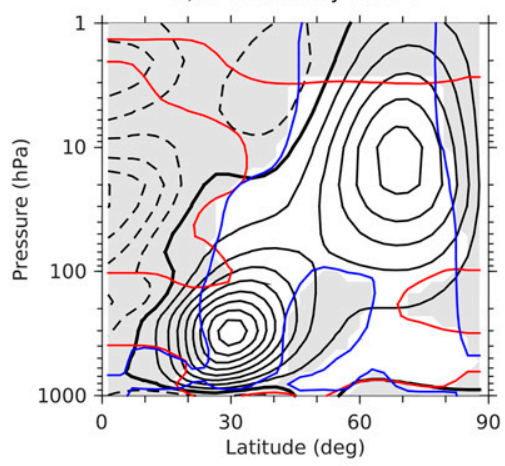

(d) $\mathrm{k} \geq 2$, experiment 4 U. $m^{\wedge} 2$ \& $\left.\right|^{\wedge} 2$ day -5 to -1

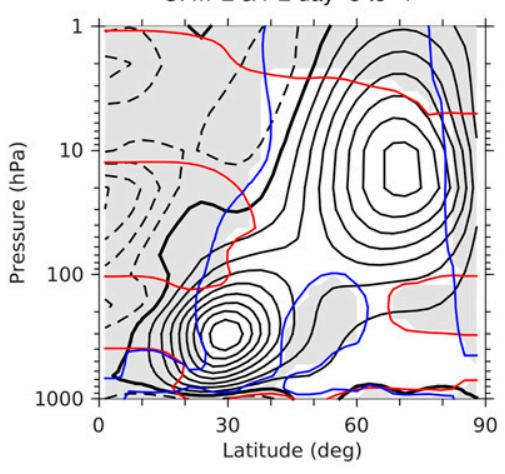

(f) $\mathrm{k} 1+\mathrm{k} \geq 2$, experiment 6 $\mathrm{U}, \mathrm{m}^{\wedge} 2$ \& $\wedge^{\wedge} 2$ day -5 to -1

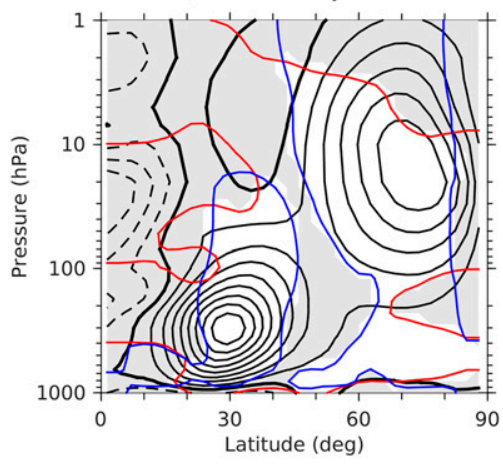

FIG. 5. (left) As in Figs. 1e and f, but for composite ensemble-mean responses for experiments (a) 1, (c) 2 and (e) 3 . The red and purple boxes denote the lag-pressure domains where the zonal-mean flow and wavenumber $k 1$ are nudged, respectively. (right) As in Figs. 1c and 1d, but for composite ensemble-mean responses for experiments (b) 1 , (d) 4 , and (f) 6 .

The importance of the higher-order wavenumbers for negative events suggests the prevailing view of downward wave coupling involving the wave-1 precursor and the zonal-mean bounded wave geometry is incomplete. As discussed in the introduction, higher-order wavenumbers may produce negative events by 1 ) generating the wave- 1 precursor, 2) modifying the zonal-mean flow, or 3) through stratospheric wave-wave interaction. Wavewave interactions would be consistent with higher-order wavenumber propagation relative to a distorted polar vortex (Smith 1983) rather than stratospheric wave-1 breaking (Palmer and Hsu 1983) since higher-order wavenumbers produce wave 1 .

Next, we consider what components control the higherorder wavenumber and zonal-mean flow responses. Nudging the wave- 1 precursor and the zonal-mean flow separately or in combination does not reproduce the higher-order wavenumber heat flux (cf. Figs. 5a,c,e with 
Fig. 1f). Nudging the wave-1 precursor shifts the vortex poleward slightly; however, the vertical reflecting surface in high latitudes does not shift below $3 \mathrm{hPa}$ and the meridional reflecting surface in midlatitudes is not reproduced (Fig. 5b). In comparison, nudging the higher-order wavenumbers produces a poleward-shifted vortex and downward-shifted reflecting surface in high latitudes but does not capture the meridional reflecting surface (Fig. 5d). Finally, nudging the wave- 1 precursor and the higherorder wavenumbers reproduces the zonal-mean flow and the bounded wave geometry (cf. Figs. $5 f$ and $1 d$ ). We note that the vertical and meridional wavenumbers in the experiments are smoother than in the CTL event because they composite over 750 ensemble members compared to 15 events.

Now that we have examined the role of the three dynamical components during negative events, we summarize their relationships. Experiment 3 shows that the wave- 1 precursor and the zonal-mean flow partially reproduce negative events but not the higher-order wavenumbers (Figs. 3c and 5e). Experiments 4-6 show that the higher-order wavenumbers are the key component reproducing negative events (Fig. 3d) and play an important role in reproducing the wave-1 precursor (Fig. 3e) and the bounded wave geometry (Fig. 5f). Therefore, we conclude that higher-order wavenumbers are the most important component producing negative events in the stratosphere.

The interpretation of the results assumes the circulation responding to the nudging is a result of the event dynamics rather than the nudging itself. However, it is possible that the nudging is providing sufficient information to constrain the circulation and the results reflect the behavior of a tightly coupled system. For example, nudging higher-order wavenumbers $(k \geq 2)$ could constrain the stratospheric wave-1 heat flux at all times since they account for a large fraction of the circulation. Therefore, there is an inherent uncertainty in quantifying the role of the dynamical components using nudging. We address this uncertainty by nudging higherorder wavenumbers to 15 randomly chosen events (experiment 7). Since the heat flux anomalies during random events differ in magnitude and sign from those during negative heat flux events by construction (cf. Figs. 4d and 6a), we normalize the anomalies by their CTL values for comparison with negative events in Fig. 6b. The results show that the spread in the responses across events is 5 times larger for random events compared to negative events. Half of the random events exhibit anomalies of the wrong sign or twice as large as the CTL events. Similar results are found when nudging waves $k 2-4$, with different random events, or when using pattern correlation to diagnose the heat flux instead of a (a) $\mathrm{k} \geq 2$, experiment 7

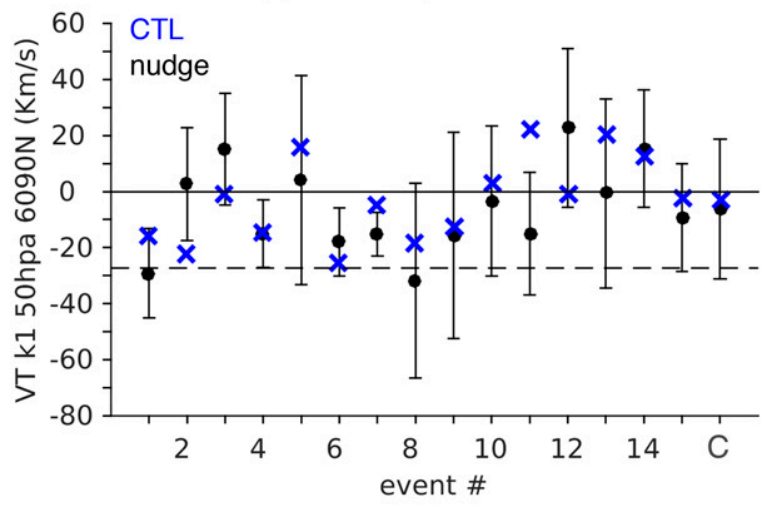

(b) $\mathrm{k} \geq 2$, experiments 4 \& 7

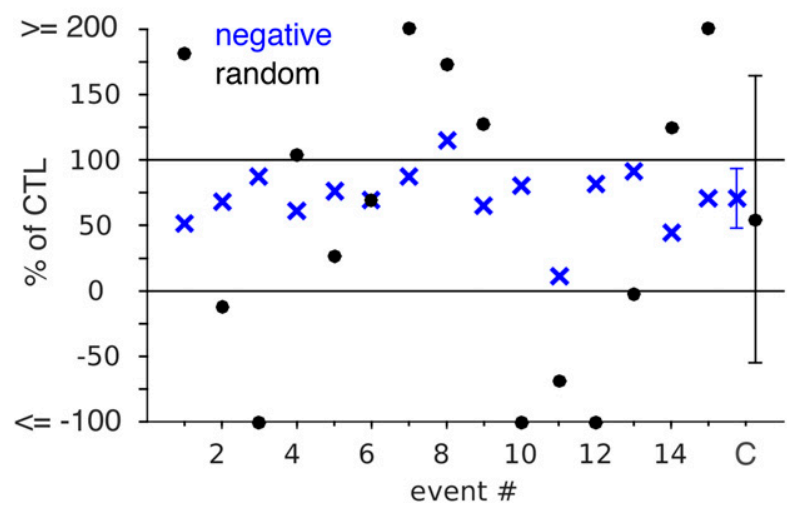

FIG. 6. (a) As in Fig. 4, but for experiment 7. (b) Ensemble-mean heat flux anomalies normalized by CTL for experiments 4 and 7 . Random and negative events are denoted in black and blue, respectively. Error bars denote the mean and one standard deviation spread across the events.

meridional average (not shown). We have performed an additional experiment where higher-order wavenumbers are nudged toward the evolution of the CTL simulation for 2000 days. Nudging wavenumbers $k \geq 2(k 2-4)$ produces a wave- 1 heat flux correlation with CTL of 0.54 (0.32) reproducing $30 \%(10 \%)$ of the variance, suggesting it is largely unconstrained. Overall, the results show higher-order wavenumbers are insufficient to constrain the wave-1 stratospheric heat flux during all times. Higher-order wavenumbers are important during specific instances corresponding to negative events consistent with the fact that they are also extreme during negative events (Figs. 1e,f).

Next, we focus on the role of the dynamical components in producing the tropospheric ingredients of negative events around the central date: the highlatitude wave-1 EP-flux convergence and geopotential height anomalies. We focus on the role of 1 ) the wave-1 precursor and the zonal-mean flow (experiment 3) because they are the two requirements for downward 
(a) $\mathrm{k} 0+\mathrm{k} 1$, experiment 3

EPFD k1 anomaly $60-90 \mathrm{~N}$

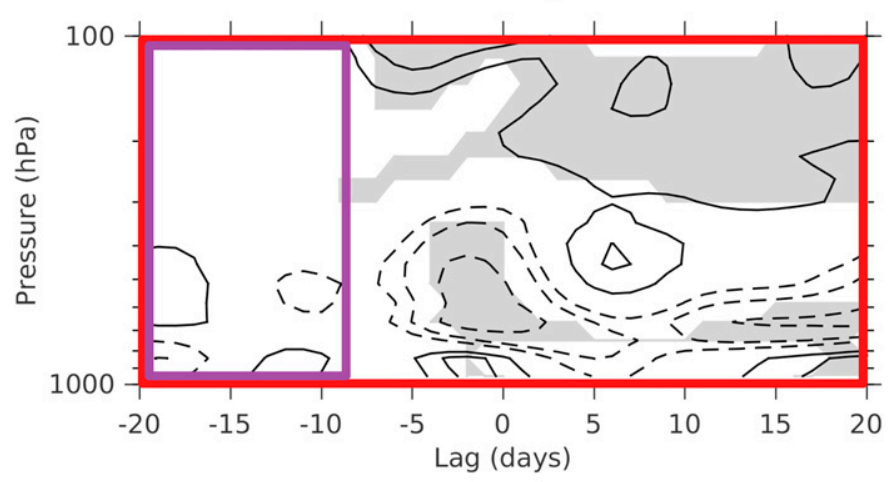

(c) $k 1+k \geq 2$, experiment 6

EPFD k1 anomaly 60-90N

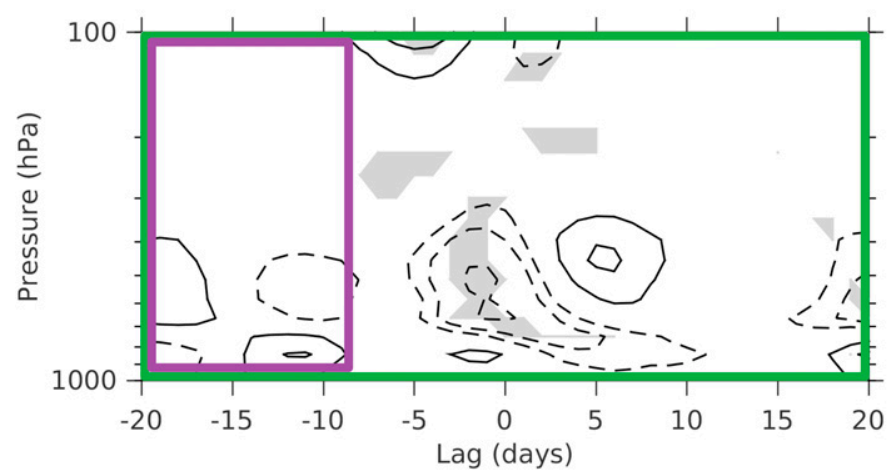

(b) $\mathrm{k} 0+\mathrm{k} 1$, experiment 3 $\mathrm{Z} \mathrm{k} 1$ anomaly $400 \mathrm{hPa}$ day -3 to +3

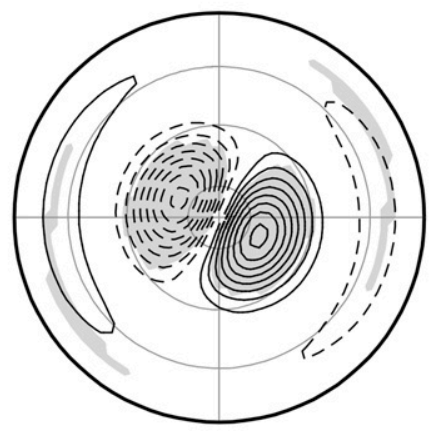

(d) $\mathrm{k} 1+\mathrm{k} \geq 2$, experiment 6 $\mathrm{Z} \mathrm{k} 1$ anomaly $400 \mathrm{hPa}$ day -3 to +3

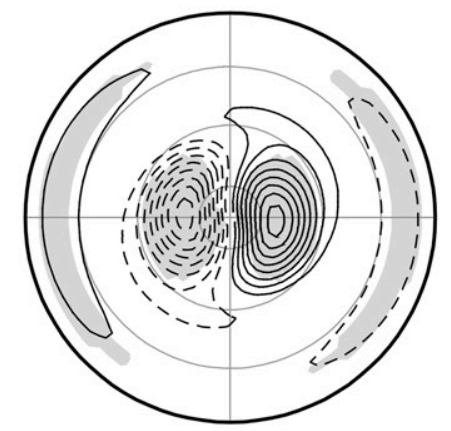

FIG. 7. As in Fig. 2, but for experiments (a),(b) 3 and (c),(d) 6. The red, purple, and green boxes denote the lagpressure domains where the zonal-mean flow and wavenumbers $k 1$ or $k \geq 2$ are nudged, respectively.

wave coupling according to the prevailing view and 2) the wave- 1 precursor and the higher-order wavenumbers (experiment 6 ) because they best reproduced the negative events in the stratosphere. Both experiments largely reproduce the tropospheric ingredients from days -3 to +3 during the composite CTL event (cf. Fig. 7 with Figs. 2c,d). We note that each of the dynamical components are not sufficient to reproduce the tropospheric ingredients alone (e.g., higher-order wavenumbers alone reproduce roughly half the amplitude; not shown). The results suggests the amplitude of the tropospheric ingredients does not simply scale with the amplitude of the stratospheric heat flux since the wave- 1 precursor and the zonal-mean flow produced a weaker heat flux response than the wave-1 precursor and the higher-order wavenumbers at $50 \mathrm{hPa}$ (Figs. 4c,f) and at $100 \mathrm{hPa}$. Understanding the relative roles of the dynamical components in determining the tropospheric response is left to future work. In summary, the results show that all three dynamical components are important for reproducing the tropospheric ingredients of negative events.
Overall, the results provide support for the prevailing view of downward wave coupling, which requires upward wave coupling (e.g., a wave-1 precursor) under specific zonal-mean-flow conditions (e.g., a bounded wave geometry). However, the answer to the first question posed in the introduction is that the prevailing view is incomplete because nudging the wave- 1 precursor alone does not reproduce negative events or the bounded wave geometry and nudging the wave- 1 precursor in combination with the zonal-mean flow only partially reproduces negative events. In contrast to the prevailing view, the results show that higher-order wavenumbers are the most important dynamical component during negative events.

\section{c. Testing the role of higher-order wavenumbers}

The previous subsection established the key role of the higher-order wavenumbers for producing negative events. However, as discussed in the second question posed in the introduction, it is unclear how they are playing a role. Higher-order wavenumbers could produce negative events by 1 ) generating the wave-1 precursor, 2) modifying the 
(a) $k 1$ climatology $+k \geq 2$ event experiment 8

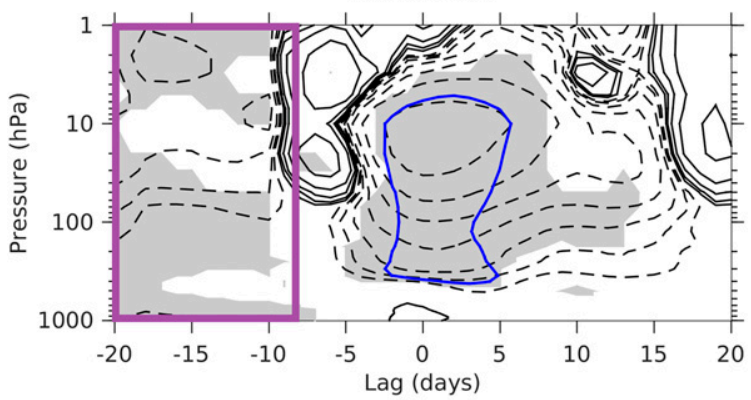

(c) k0 climatology $+k \geq 2$ event experiment 9

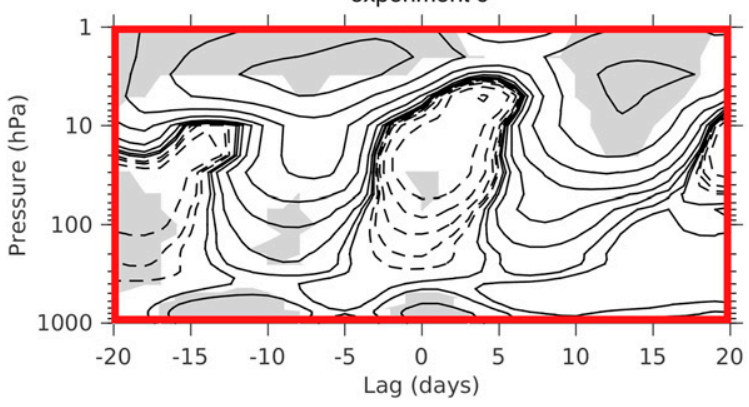

(e) $k 0+k 1$ climatology $+k \geq 2$ event experiment 10

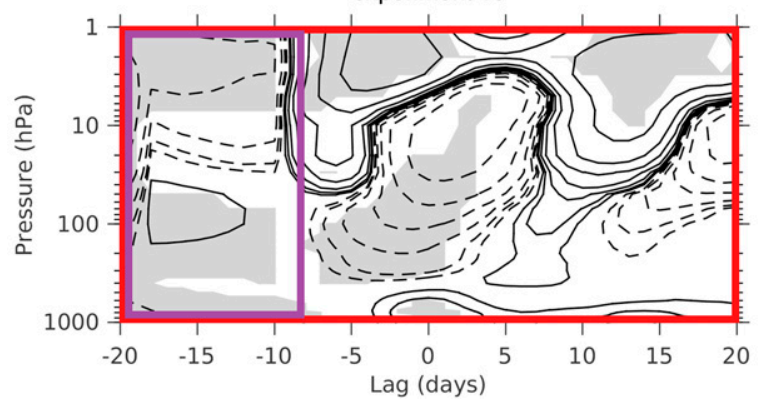

(b) k1 climatology $+k \geq 2$ event experiment $8(71 \%)$

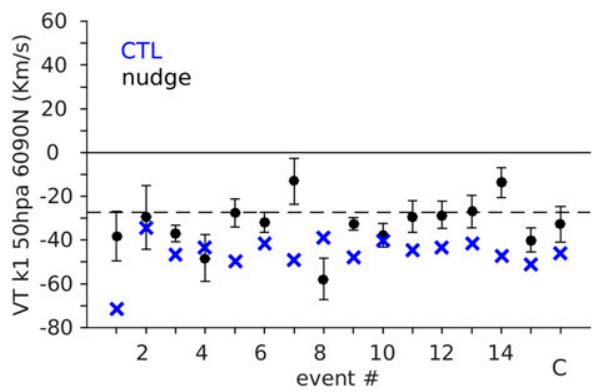

(d) k0 climatology $+k \geq 2$ event experiment $9(26 \%)$

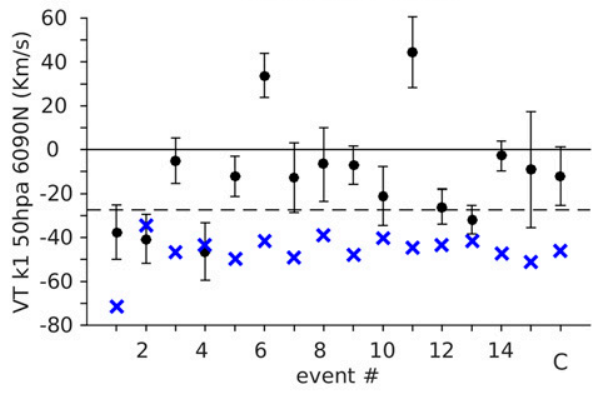

(f) $k 0+k 1$ climatology $+k \geq 2$ event experiment $10(38 \%)$

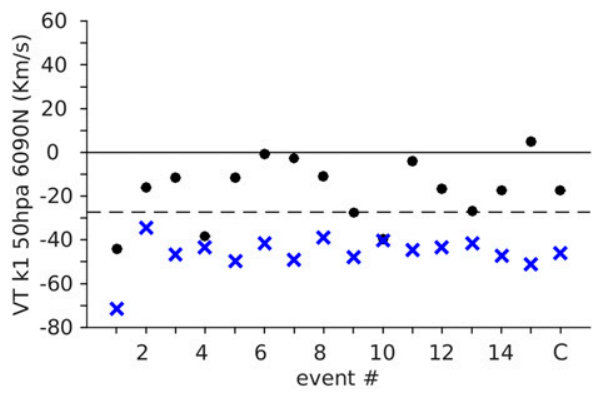

Fig. 8. As in (a),(c),(e) Figs. 1a and 1b and (b),(d),(f) Fig. 4, but for experiments (top) 8, (middle) 9, and (bottom) 10. The red and purple boxes denote the lag-pressure domains where the zonal-mean flow and wavenumber $k 1$ are nudged, respectively. Boxes denoting the lag-pressure domain where wavenumbers $k \geq 2$ are nudged are omitted for clarity. Error bars in (f) are small since all wavenumbers are nudged.

zonal-mean flow, or 3) through stratospheric wave-wave interactions. In this section we test roles 1 and 2 above by performing mechanism denial experiments where we nudge the higher-order wavenumbers as before (experiment 4) but prevent changes in the wave-1 precursor or the zonal-mean flow by nudging the components to the timeindependent climatology (experiments 8-10). If the higher-order wavenumbers reproduce negative events via the wave- 1 precursor or the zonal-mean flow, the heat flux response in experiments $8-10$ will be reduced relative to experiment 4 .

Nudging the wave-1 precursor to wave-1 climatology does not reduce the magnitude of the stratospheric heat flux anomalies around day 0 in comparison to the experiments where the wave-1 precursor is changing, since both experiments reproduce $70 \%$ of the composite CTL heat flux anomalies at $50 \mathrm{hPa}$ (cf. Figs. 8a,b with Figs. $3 d$ and $4 d$ ). We note that the wave-1 heat flux anomalies are not exactly zero when nudged to climatology because the climatological meridional wind $v^{\prime}$ and temperature $T^{\prime}$ are nudged instead of the climatological $v^{\prime} T^{\prime}$. However, the nudging successfully eliminates the anomalous positive stratospheric heat fluxes associated with the wave-1 precursor (Fig. A1b). Nudging the zonal-mean flow to climatology greatly reduces the magnitude of the heat flux anomalies around day 0 in 


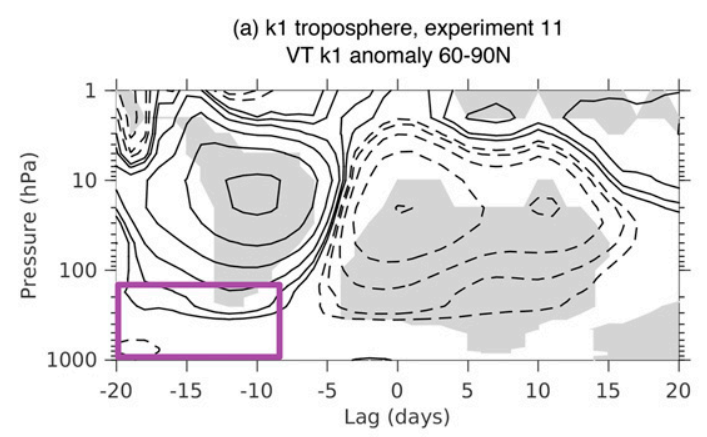

(c) k1 troposphere, experiment 11 $U, m^{\wedge} 2 \& I^{\wedge} 2$ day -5 to -1

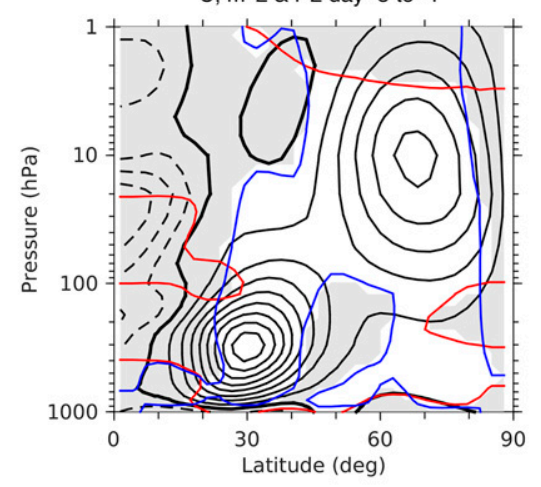

(e) k1 troposphere, experiment 11 VT $\mathrm{k} \geq 2$ anomaly $40-70 \mathrm{~N}$

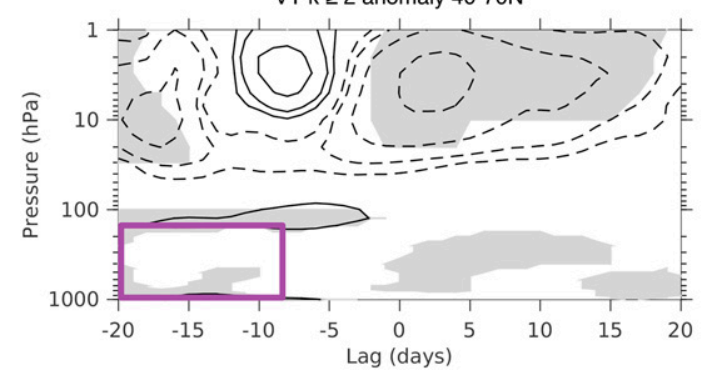

(b) $k 1+k \geq 2$ troposphere, experiment 12 VT k1 anomaly $60-90 \mathrm{~N}$

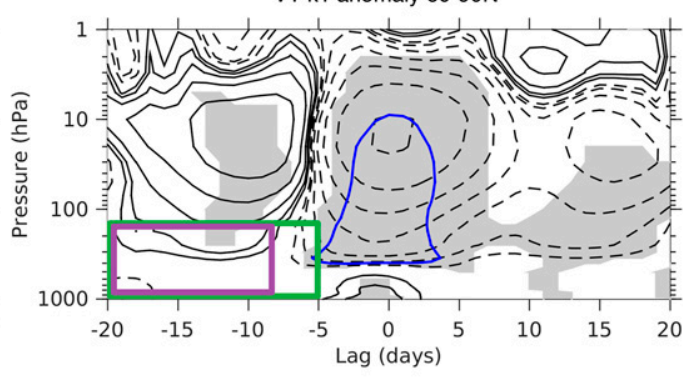

(d) $k 1+k \geq 2$ troposphere, experiment 12 $U, m^{\wedge} 2$ \& ${ }^{\wedge} 2$ day -5 to -1

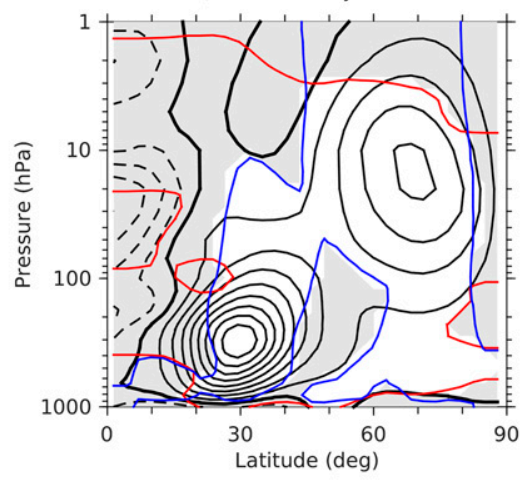

(f) $k 1+k \geq 2$ troposphere, experiment 12

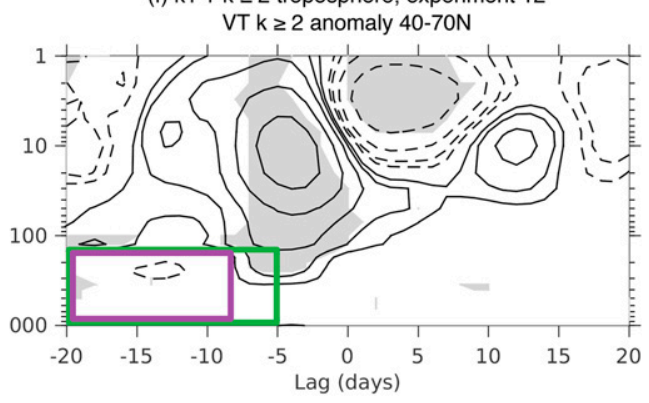

FIG. 9. As in Fig. 1, but for experiments (a),(c),(e) 11 and (b),(d),(f) 12. The purple and green boxes denote the lag-pressure domain where wavenumbers $k 1$ and $k \geq 2$ are nudged, respectively.

comparison to the experiments where the zonal-mean flow is changing (cf. Figs. 8c,d with Figs. $3 d$ and $4 d$ ). In particular, the heat flux anomalies are reduced across most events and the composite anomalies are reduced from $70 \%$ to $26 \%$ of the composite CTL event. Finally, nudging the zonal-mean flow and the wave- 1 precursor to climatology also reduces the magnitude of the heat flux anomalies around day 0 in comparison the experiments where the components are changing (cf. Figs. 8e,f with Figs. 3d and 4d). The composite anomalies are reduced from $70 \%$ to $38 \%$ of the composite CTL event. Similar results are found when nudging the wave-1 precursor and zonal-mean flow to climatology and wavenumbers $k 2-4$ to the event evolution (Fig. A2b).
The results suggest the higher-order wavenumbers control negative events through the zonal-mean flow. This is analogous to the role of higher-order wavenumbers in sharpening the vortex edge (McIntyre 1982) except that for negative events the higher-order wavenumbers shape the bounded wave geometry. However, higher-order wavenumbers must be doing more than modifying the zonal-mean flow since nudging the higher-order wavenumbers alone better reproduces negative events than nudging the zonal-mean flow alone (Figs. 3b,d). Therefore, we conclude that higher-order wavenumbers also produce negative events through stratospheric wave-wave interaction (Smith 1983). Thus, the answer to the second question posed in the introduction is that the higher-order wavenumbers 
(a) $\mathrm{k} 1+\mathrm{k} \geq 2$ troposphere, experiment 12 EPFD k1 anomaly $60-90 \mathrm{~N}$

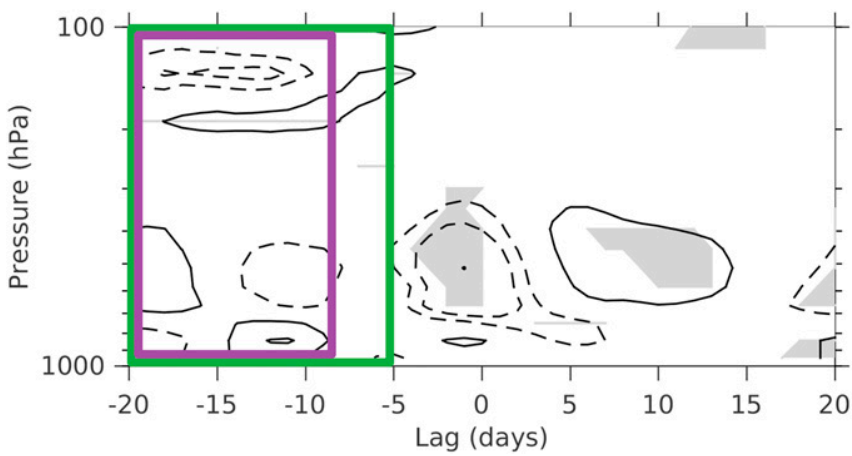

(b) $k 1+k \geq 2$ troposphere, experiment 12 $\mathrm{Z} \mathrm{k} 1400 \mathrm{hPa}$ anomaly day -3 to +3



FIG. 10. As in Fig. 2, but for experiment 12.

play a role during negative events by modifying the zonal-mean flow and through stratospheric wave-wave interaction.

\section{d. Quantifying the role of upward wave coupling}

The third question posed in the introduction is whether upward wave coupling plays a role during negative events. Upward wave coupling from a tropospheric wave source may produce downward wave coupling according to the prevailing view. Conversely, downward wave coupling may result from a stratospheric wave source. In this subsection we quantify the role of upward coupling during negative events by nudging the tropospheric wave-1 precursor alone (experiment 11) and in combination with the tropospheric higher-order wavenumber precursor (experiment 12). If upward wave coupling plays a role, then the tropospheric wave precursors should reproduce the stratospheric wave precursors and the stratospheric and tropospheric ingredients during negative events.

We begin by examining whether the tropospheric wave precursors can reproduce the stratospheric ingredients. Nudging the tropospheric wave-1 precursor reproduces the stratospheric wave- 1 heat flux precursor but does not reproduce the stratospheric higherorder wavenumber heat flux precursor, the wave-1 heat flux reversal, or the change in the zonal-mean flow involving the downward-shifted reflecting surface and meridional reflecting surface (cf. Figs. 9a,c,e with Figs. 1b,d,f). Conversely, nudging the tropospheric wave-1 and higher-order wavenumber precursors reproduces the stratospheric wave- 1 and higher-order wavenumber heat flux precursors, as well as the wave-1 heat flux reversal, and better captures the change in the zonal-mean flow (cf. Figs. 9b,d,f with Figs. 1b,d,f). Specifically, it accounts for $73 \%$ of the composite CTL event high-latitude wave- 1 heat flux anomaly at $50 \mathrm{hPa}$ averaged from days -3 to +3 (blue $\times$ symbols in Fig. 11). Similar results are found when nudging the tropospheric wave- 1 and wavenumber $k 2-4$ precursors (Figs. A2c,d) or when nudging the tropospheric wave-1 and higher-order wavenumber precursors from days -20 to -8 (not shown). Since nudging all tropospheric wave precursors largely reproduced the stratospheric ingredients, next we focus on whether they capture the tropospheric ingredients. Nudging all the tropospheric wave precursors largely captures the highlatitude tropospheric wave-1 EP-flux convergence and geopotential height anomalies from days -3 to +3 (cf. Fig. 10 with Figs. 2c,d).

The results show the tropospheric wave precursors capture the overall stratospheric and tropospheric evolution of negative events. However, it is unclear whether the response is a result of the event dynamics or simply due to nudging the troposphere prior to the event. To determine whether the events are more predictable than the general case, we compare our results with an experiment nudging the tropospheric

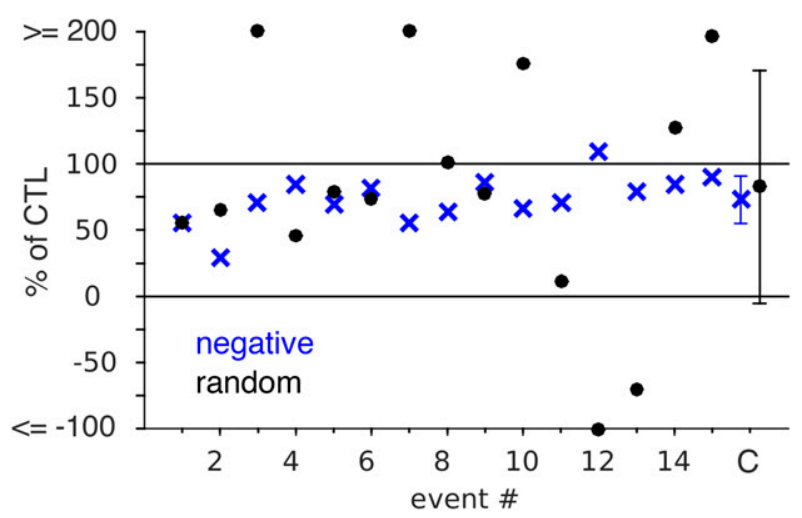

FIG. 11. As in Fig. 6b, but for experiments 12 and 13. 
(a) $k \geq 1$ stratosphere day -20 to +20 , experiment 14 EPFD $k 1$ anomaly $60-90 \mathrm{~N}$

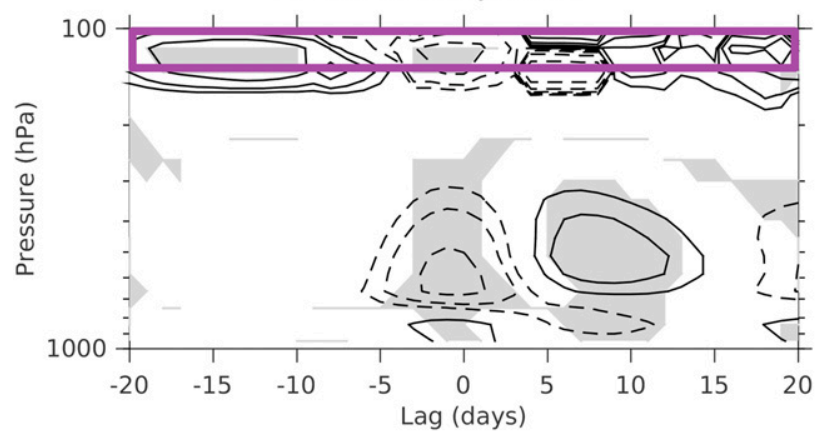

(c) $k \geq 1$ stratosphere day -5 to +5 , experiment 15 EPFD k1 anomaly 60-90N

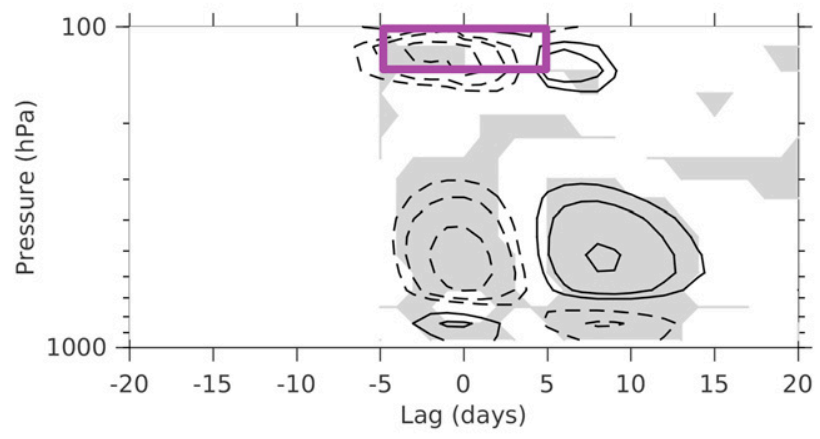

(e) k1 stratosphere day -20 to +20 , experiment 16 EPFD k1 anomaly $60-90 \mathrm{~N}$

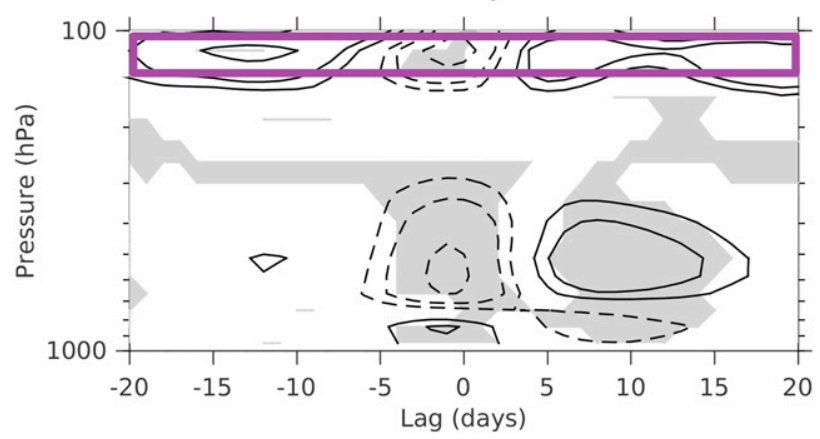

(b) $k \geq 1$ stratosphere day -20 to +20 , experiment 14 $\mathrm{Z} \mathrm{k1}$ anomaly $400 \mathrm{hPa}$ day -3 to +3

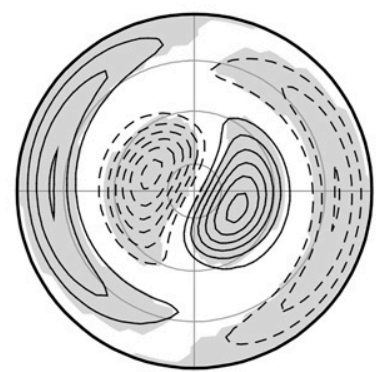

(d) $\mathrm{k} \geq 1$ stratosphere day -5 to +5 , experiment 15 $\mathrm{Zk} 1$ anomaly $400 \mathrm{hPa}$ day -3 to +3

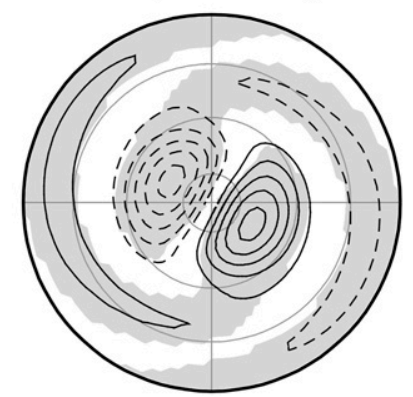

(f) $\mathrm{k} 1$ stratosphere day -20 to +20 , experiment 16 $\mathrm{Zk} 1$ anomaly $400 \mathrm{hPa}$ day -3 to +3

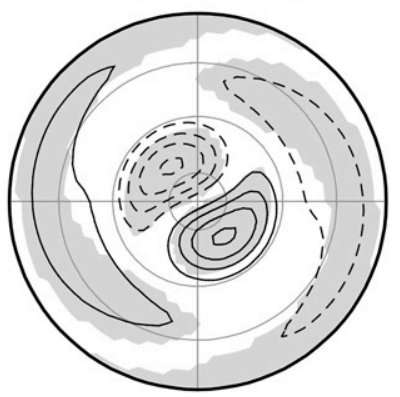

FIG. 12. As in Fig. 2, but for experiments (a),(b) 14, (c),(d) 15, and (e),(f) 16. Purple box denotes the lag-pressure domain where wavenumbers $k 1$ or $k \geq 1$ are nudged.

wave precursors during 15 random events (experiment 13). The results show that the spread in the normalized stratospheric heat flux responses across events is 5 times larger during random events compared to negative events (Fig. 11). Certain random events produce responses that are of the wrong sign or twice as large as the CTL events. Similar results are found when nudging tropospheric wave precursors $k 1-4$, with different random events or using pattern correlation to diagnose the heat flux instead of a meridional average (not shown). The results suggest that the wave-1 heat flux is more predictable during negative events than in the general case. Consistently, we confirm that the stratospheric and tropospheric ingredients are not the result of the persistence of the initial condition since most of the memory of the initial condition is lost by day 0 (not shown).

In summary, nudging all tropospheric wave precursors shows they are the source of the stratospheric wave precursors and largely reproduces the stratospheric and tropospheric ingredients of negative events. Thus, the answer to the third question posed in the introduction is 
that upward wave coupling plays a role prior to negative events consistent with the prevailing view of downward wave coupling. However, the prevailing view is incomplete because upward wave coupling involving both wave- 1 and the higher-order wavenumbers is necessary to capture negative events.

\section{e. Testing the causal role of the stratosphere on the troposphere}

The fourth and final question posed in the introduction is whether the stratosphere plays a causal role during negative events. Thus far, none of the experiments have demonstrated that the stratosphere produces the tropospheric ingredients during negative events since negative eddy heat fluxes do not necessarily imply downward wave coupling (Plumb 2010). Therefore in this subsection, we explicitly test the role of the stratosphere by performing three experiments: the first nudges the stratospheric wave evolution (wavenumbers $k \geq 1$ ) over the entire life cycle (days -20 to +20 , experiment 14 ), the second nudges the stratospheric waves during the period when the stratospheric heat flux becomes negative (days -5 to +5 , experiment 15 ), while the third nudges the stratospheric wave-1 evolution (days -20 to +20 , experiment 16). If the stratosphere plays a causal role, then nudging the stratospheric wave evolution should reproduce the tropospheric ingredients during negative events.

Nudging the stratospheric wave evolution largely reproduces the high-latitude tropospheric wave-1 EPflux convergence and geopotential height anomalies on days -3 to +3 of the negative events (cf. Figs. 12a,b with Figs. 2c,d). Similar results are found when nudging stratospheric wavenumbers $k 1-4$ (Figs. A2e,f) or when nudging the stratospheric waves only during days -5 to +5 (cf. Figs. 12c,d with Figs. 2c,d). Similar but smaller-magnitude results are found when nudging the stratospheric wave-1 evolution throughout the event (cf. Figs. 12e,f with Figs. 2c,d). Understanding the relative roles of wave-1 and higher-order wavenumbers in determining the tropospheric response is left to future work.

Overall, the experiments show that nudging the stratospheric wave evolution largely reproduces the tropospheric ingredients during negative events. Therefore, the answer to the fourth question posed in the introduction is that the stratosphere plays a causal role during negative events. The results provide further support for the prevailing view of downward wave coupling because stratospheric wave 1 produces a tropospheric wave- 1 response. In addition, an EP-flux budget analysis shows that the transient evolution of wave-1 activity is consistent with the creation of stratospheric EP-flux divergence, for example, EP-flux convergence driven by an increase in wave activity as it enters the high-latitude stratosphere, $\nabla \cdot \mathbf{F}=-\partial A / \partial t<0$, followed by EP-flux divergence driven by a decrease of wave activity as it exits the stratosphere and enters the troposphere, $\nabla \cdot \mathbf{F}=-\partial A / \partial t>0$ (not shown). However once again, the prevailing view is incomplete because nudging all stratospheric waves better captures the tropospheric ingredients than does nudging wave 1 alone.

\section{Conclusions and discussion}

\section{a. Conclusions}

Extreme stratospheric negative wave-1 heat flux events couple with the troposphere via an anomalous wave-1 signal. The goal of this study was to understand the dynamics of negative events, which are not associated with a weak vortex, using a dry dynamical core model. Ensemble spectral nudging experiments were performed to isolate the role of specific dynamical components during the events: the wave- 1 precursor, the stratospheric zonal-mean flow, and the higher-order wavenumbers. The answers to the questions posed in the introduction are as follows:

1) What is the role of the wave-1 precursor and the zonal-mean flow?

The results provide support for the prevailing view of downward wave coupling involving upward wave coupling (e.g., a wave-1 precursor) under specific zonal-mean-flow conditions (e.g., bounded wave geometry), resulting in wave reflection (Harnik and Lindzen 2001; Perlwitz and Harnik 2003, 2004; Harnik 2009; Shaw et al. 2010, 2011; Lubis et al. 2016). However, the prevailing view is incomplete because nudging the wave-1 precursor alone does not reproduce the negative events or the bounded wave geometry (Figs. $3 a$ and $5 b$ ) and nudging the wave-1 precursor in combination with the zonalmean flow captures only $37 \%$ of the event amplitude in the midstratosphere (Figs. 3c and 4c).

2) What is the role of higher-order planetary wavenumbers?

Stratospheric higher-order wavenumbers are extreme prior to negative events in the model and reanalysis (Figs. 1e,f). They are the most important dynamical component during negative events in the model (Figs. 3a, b,d) capturing $70 \%$ of the event amplitude in the midstratosphere (Fig. 4c). The results suggest they contribute to the event evolution by modifying the zonal-mean flow and through wave-wave interaction 
(Figs. 3b,d and 8c). The former is analogous to sharpening the vortex edge before stratospheric sudden warmings (McIntyre 1982) while the latter is consistent with higher-order wavenumbers propagating on a distorted polar vortex (Smith 1983).

3) What is the role of upward wave coupling?

Nudging all tropospheric wave precursors confirms they are the source of the stratospheric wave precursors and largely reproduces the stratospheric and tropospheric ingredients of negative events (Figs. 9b, $\mathrm{d}$,f and 10). The results are consistent with the prevailing view of downward wave coupling following from upward wave coupling. However, the prevailing view is incomplete because the upward wave-1 coupling is insufficient to capture negative events (Figs. 9a,c,e).

4) Does the stratosphere play a causal role?

Nudging the stratospheric wave evolution largely reproduces the tropospheric ingredients during negative events (Figs. 12a-d), showing the stratosphere plays a causal role. However, nudging the stratospheric wave-1 evolution alone produces a smaller tropospheric response (Figs. 12e,f), suggesting the prevailing view of downward wave coupling is incomplete.

\section{b. Discussion}

The results suggest the prevailing view of downward wave coupling in the literature should be revised to include a key role for higher-order wavenumbers. However, further research is required to understand the detailed role of the higher-order wavenumbers. In particular, the degree of wave-wave interaction has not been quantified and it is unclear why the higher-order wavenumbers play a dominant role during negative events. The latter could be because higher-order wavenumbers control the events under specific zonal-meanflow conditions (e.g., bounded wave geometry) or because they are extreme themselves during the events.

The results suggest negative events are predictable several days in advance. Subseasonal hindcasts from models participating in the Subseasonal-to-Seasonal (S2S) prediction project (Vitart et al. 2017) could be used to 1) assess the predictability of negative events and the poleward shift of the Atlantic jet and 2) determine whether initializing the tropospheric wave precursors provides enhanced predictive skill. This will be explored in a future study.

Finally, Dunn-Sigouin and Shaw (2015) showed that extreme stratospheric positive wave-1 heat flux events, defined by heat flux values exceeding the 95 th percentile, also couple with the troposphere via an anomalous wave- 1 signal. The relative contributions of the different dynamical mechanisms during positive events is the subject of ongoing work.

Acknowledgments. The authors thank Karen Smith, Isla Simpson, Richard Seager, Mingfang Ting, Lorenzo Polvani, and Peter Hitchcock for helpful discussions. They also thank Ed Gerber and two anonymous reviewers whose comments improved the manuscript. Both authors are supported by the National Science Foundation (AGS-1129519). EDS also acknowledges support from the Natural Sciences and Engineering Research Council of Canada.

\section{APPENDIX}

\section{Additional Diagnostics and Supporting Results}

Shown below are additional diagnostics for experiments 4 and 8 (Fig. A1) and supporting results from experiments 17-20 (Fig. A2).

(a) $k \geq 2$ event, experiment 4

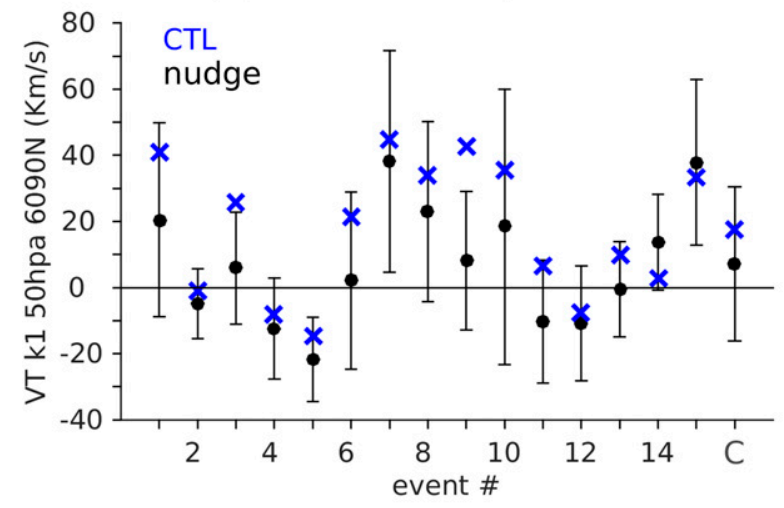

(b) $k 1$ climatology $+k \geq 2$ event, experiment 8

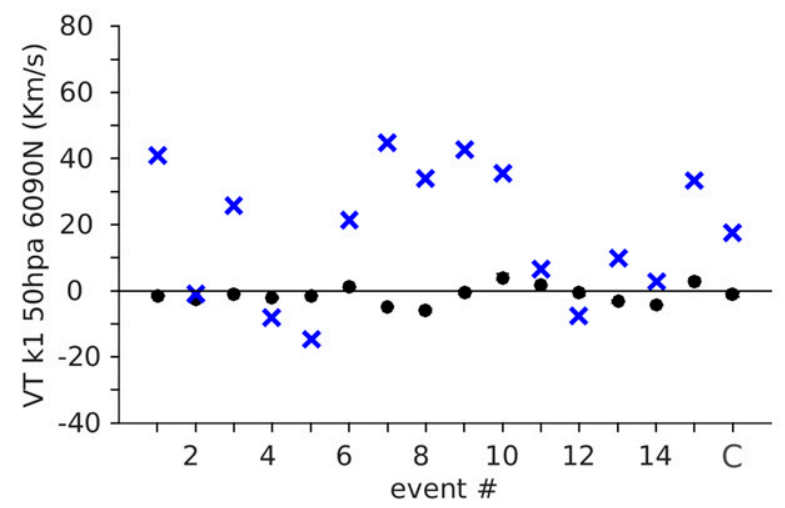

FIG. A1. As in Fig. 4, but for anomalous high-latitude wave-1 heat flux at $50 \mathrm{hPa}$ averaged over days -13 to -8 for experiments (a) 4 and (b) 8 . 

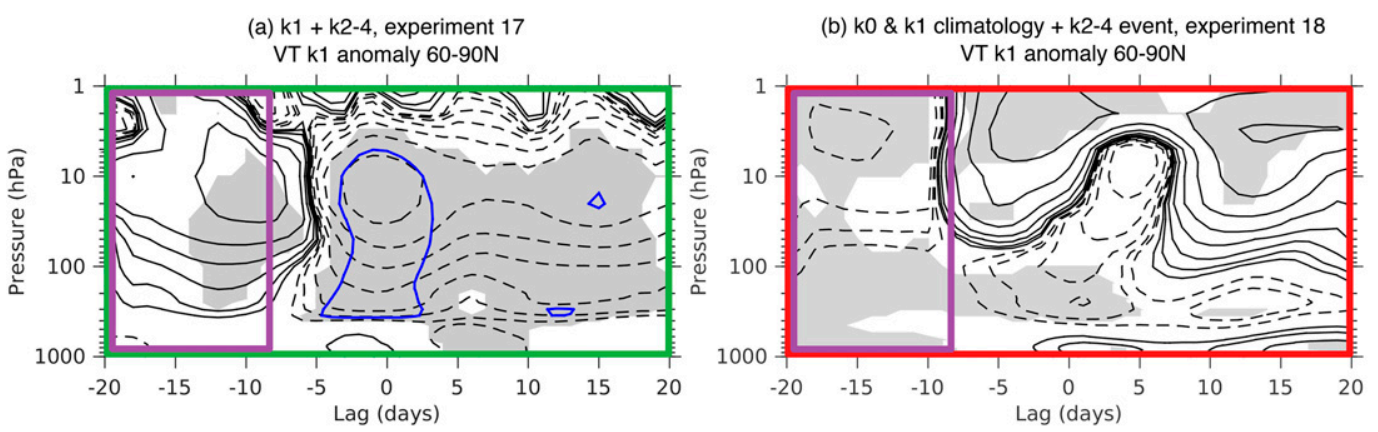

(c) k1 + k2-4 troposphere, experiment 19 VT k1 anomaly 60-90N

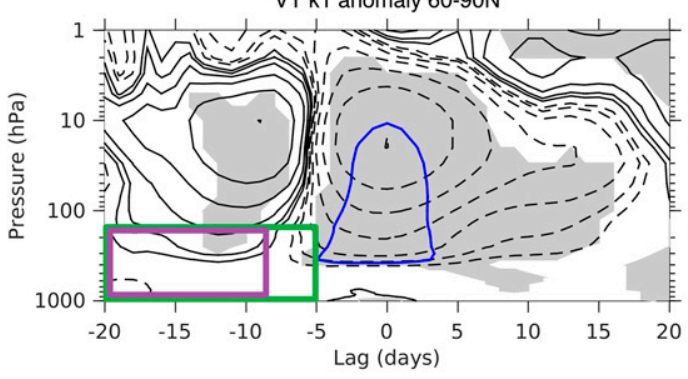

(d) $\mathrm{k} 1+\mathrm{k} 2-4$ troposphere, experiment 19

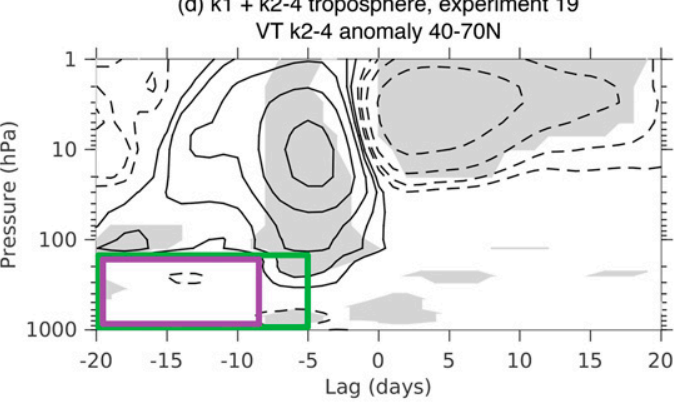

(e) k1-4 stratosphere day -20 to +20 , experiment 20 EPFD k1 anomaly $60-90 \mathrm{~N}$

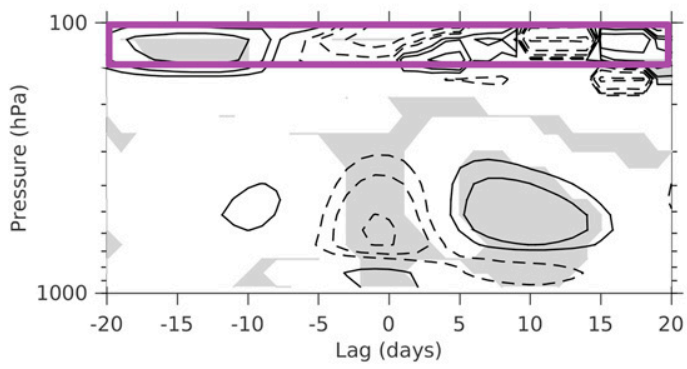

(f) k1-4 stratosphere day -20 to +20 , experiment 20 Z k1 anomaly $400 \mathrm{hPa}$ day -3 to +3

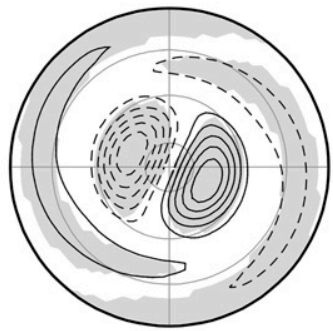

FIG. A2. (a) As in Fig. 3f, but for experiment 17. (b) As in Fig. 8e, but for experiment 18. (c),(d) As in Figs. 9b and 9f, respectively, but for experiment 19. (e),(h) As in Figs. 12a and 12b, respectively, but for experiment 20.

\section{REFERENCES}

Andrews, D. G., J. R. Holton, and C. B. Leovy, 1987: Middle Atmosphere Dynamics. International Geophysics Series, Vol. 40, Academic Press, 489 pp.

Baldwin, M. P., and T. J. Dunkerton, 2001: Stratospheric harbingers of anomalous weather regimes. Science, 294, 581-584, https://doi.org/10.1126/science.1063315.

Dee, D., and Coauthors, 2011: The ERA-Interim reanalysis: Configuration and performance of the data assimilation system. Quart. J. Roy. Meteor. Soc., 137, 553-597, https://doi.org/ 10.1002/qj.828.

Douville, H., 2009: Stratospheric polar vortex influence on Northern Hemisphere winter climate variability. Geophys. Res. Lett., 36, L18703, https://doi.org/10.1029/2009GL039334.

Dunn-Sigouin, E., and T. A. Shaw, 2015: Comparing and contrasting extreme stratospheric events, including their coupling to the tropospheric circulation. J. Geophys. Res. Atmos., 120 1374-1390, https://doi.org/10.1002/2014JD022116.

Gerber, E. P., and L. M. Polvani, 2009: Stratosphere-troposphere coupling in a relatively simple AGCM: The importance of stratospheric variability. J. Climate, 22, 1920-1933, https:// doi.org/10.1175/2008JCLI2548.1.

, and Coauthors, 2010: Stratosphere-troposphere coupling and annular mode variability in chemistry-climate models. J. Geophys. Res., 115, D00M06, https://doi.org/10.1029/2009JD013770.

Greatbatch, R. J., G. Gollan, T. Jung, and T. Kunz, 2012: Factors influencing Northern Hemisphere winter mean atmospheric circulation anomalies during the period 1960/61 to 2001/02. Quart. J. Roy. Meteor. Soc., 138, 1970-1982, https://doi.org/ 10.1002/qj.1947.

Hansen, F., R. J. Greatbatch, G. Gollan, T. Jung, and A. Weisheimer, 2017: Remote control of North Atlantic oscillation predictability via the stratosphere. Quart. J. Roy. Meteor. Soc., 143, 706-719, https://doi.org/10.1002/qj.2958.

Harnik, N., 2009: Observed stratospheric downward reflection and its relation to upward pulses of wave activity. J. Geophys. Res., 114, D08120, https://doi.org/10.1029/2008JD010493. , and R. S. Lindzen, 2001: The effect of reflecting surfaces on the vertical structure and variability of stratospheric planetary waves. J. Atmos. Sci., 58, 2872-2894, https://doi.org/10.1175/ 1520-0469(2001)058<2872:TEORSO >2.0.CO;2. 
Held, I. M., and M. J. Suarez, 1994: A proposal for the intercomparison of the dynamical cores of atmospheric general circulation models. Bull. Amer. Meteor. Soc., 75, 1825-1830, https://doi.org/ 10.1175/1520-0477(1994)075<1825:APFTIO > 2.0.CO;2.

Hitchcock, P., and I. R. Simpson, 2014: The downward influence of stratospheric sudden warmings. J. Atmos. Sci., 71, 3856-3876, https://doi.org/10.1175/JAS-D-14-0012.1.

- and P. H. Haynes, 2016: Stratospheric control of planetary waves. Geophys. Res. Lett., 43, $11884-11892$, https://doi.org/ 10.1002/2016GL071372.

— , T. G. Shepherd, and G. L. Manney, 2013: Statistical characterization of Arctic polar-night jet oscillation events. J. Climate, 26, 2096-2116, https://doi.org/10.1175/JCLI-D-12-00202.1.

Kidston, J., A. A. Scaife, S. C. Hardiman, D. M. Mitchell, N. Butchart, M. P. Baldwin, and L. J. Gray, 2015: Stratospheric influence on tropospheric jet streams, storm tracks and surface weather. Nat. Geosci., 8, 433-440, https://doi.org/10.1038/ ngeo2424.

Kodera, K., H. Mukougawa, P. Maury, M. Ueda, and C. Claud, 2016: Absorbing and reflecting sudden stratospheric warming events and their relationship with tropospheric circulation. J. Geophys. Res. Atmos., 121, 80-94, https://doi.org/10.1002/ 2015JD023359.

Limpasuvan, V., D. W. Thompson, and D. L. Hartmann, 2004: The life cycle of the Northern Hemisphere sudden stratospheric warmings. J. Climate, 17, 2584-2596, https://doi.org/10.1175/ 1520-0442(2004)017<2584:TLCOTN $>2.0$.CO;2.

—, D. L. Hartmann, D. W. J. Thompson, K. Jeev, and Y. L. Yung, 2005: Stratosphere-troposphere evolution during polar vortex intensification. J. Geophys. Res., 110, D24101, https:// doi.org/10.1029/2005JD006302.

Lubis, S. W., K. Matthes, N.-E. Omrani, N. Harnik, and S. Wahl, 2016: Influence of the quasi-biennial oscillation and sea surface temperature variability on downward wave coupling in the Northern Hemisphere. J. Atmos. Sci., 73, 1943-1965, https://doi.org/10.1175/JAS-D-15-0072.1.

_- V. Silverman, K. Matthes, N. Harnik, N.-E. Omrani, and S. Wahl, 2017: How does downward planetary wave coupling affect polar stratospheric ozone in the Arctic winter stratosphere? Atmos. Chem. Phys., 17, 2437-2458, https://doi.org/ 10.5194/acp-17-2437-2017.

McIntyre, M. E., 1982: How well do we understand the dynamics of stratospheric warmings? J. Meteor. Soc. Japan, 60, 37-65, https://doi.org/10.2151/jmsj1965.60.1_37.

McLandress, C., and T. G. Shepherd, 2009: Impact of climate change on stratospheric sudden warmings as simulated by the Canadian Middle Atmosphere Model. J. Climate, 22, 54495463, https://doi.org/10.1175/2009JCLI3069.1.

Mukougawa, H., S. Noguchi, Y. Kuroda, R. Mizuta, and K. Kodera, 2017: Dynamics and predictability of downwardpropagating stratospheric planetary waves observed in March 2007. J. Atmos. Sci., 74, 3533-3550, https://doi.org/10.1175/ JAS-D-16-0330.1.

Palmer, T., and C. F. Hsu, 1983: Stratospheric sudden coolings and the role of nonlinear wave interactions in preconditioning the circumpolar flow. J. Atmos. Sci., 40, 909-928, https://doi.org/ 10.1175/1520-0469(1983)040<0909:SSCATR >2.0.CO;2.

Perlwitz, J., and N. Harnik, 2003: Observational evidence of a stratospheric influence on the troposphere by planetary wave reflection. J. Climate, 16, 3011-3026, https://doi.org/10.1175/ 1520-0442(2003)016<3011:OEOASI >2.0.CO;2.

, and - 2004: Downward coupling between the stratosphere and troposphere: The relative roles of wave and zonal mean processes. J. Climate, 17, 4902-4909, https://doi.org/ 10.1175/JCLI-3247.1.

Plumb, R. A., 2010: Planetary waves and the extratropical winter stratosphere. The Stratosphere: Dynamics, Transport and Chemistry, Geophys. Monogr., Vol. 190, Amer. Geophys. Union, 23-41.

Polvani, L. M., and P. J. Kushner, 2002: Tropospheric response to stratospheric perturbations in a relatively simple general circulation model. Geophys. Res. Lett., 29, 1114, https://doi.org/ 10.1029/2001GL014284.

, and D. W. Waugh, 2004: Upward wave activity flux as a precursor to extreme stratospheric events and subsequent anomalous surface weather regimes. J. Climate, 17, 3548-3554, https://doi.org/ 10.1175/1520-0442(2004)017<3548:UWAFAA > 2.0.CO;2.

Randel, W. J., 1987: A study of planetary waves in the southern winter troposphere and stratosphere. Part I: Wave structure and vertical propagation. J. Atmos. Sci., 44, 917-935, https://doi.org/ 10.1175/1520-0469(1987)044<0917:ASOPWI>2.0.CO;2.

Scaife, A., and Coauthors, 2016: Seasonal winter forecasts and the stratosphere. Atmos. Sci. Lett., 17, 51-56, https://doi.org/ 10.1002/asl.598.

Scinocca, J. F., and P. H. Haynes, 1998: Dynamical forcing of stratospheric planetary waves by tropospheric baroclinic eddies. J. Atmos. Sci., 55, 2361-2392, https://doi.org/10.1175/ 1520-0469(1998)055<2361:DFOSPW >2.0.CO;2.

Shaw, T. A., and J. Perlwitz, 2013: The life cycle of Northern Hemisphere downward wave coupling between the stratosphere and troposphere. J. Climate, 26, 1745-1763, https:// doi.org/10.1175/JCLI-D-12-00251.1.

- , and - 2014: On the control of the residual circulation and stratospheric temperatures in the Arctic by planetary wave coupling. J. Atmos. Sci., 71, 195-206, https://doi.org/10.1175/ JAS-D-13-0138.1.

,-- , and N. Harnik, 2010: Downward wave coupling between the stratosphere and troposphere: The importance of meridional wave guiding and comparison with zonal-mean coupling. J. Climate, 23, 6365-6381, https://doi.org/10.1175/ 2010JCLI3804.1.

,,,--- P. A. Newman, and S. Pawson, 2011: The impact of stratospheric ozone changes on downward wave coupling in the Southern Hemisphere. J. Climate, 24, 4210-4229, https:// doi.org/10.1175/2011JCLI4170.1.

Sigmond, M., J. Scinocca, V. Kharin, and T. Shepherd, 2013: Enhanced seasonal forecast skill following stratospheric sudden warmings. Nat. Geosci., 6, 98-102, https://doi.org/10.1038/ngeo1698.

Smith, A. K., 1983: Observation of wave-wave interactions in the stratosphere. J. Atmos. Sci., 40, 2484-2496, https://doi.org/ 10.1175/1520-0469(1983)040<2484:OOWWII >2.0.CO;2.

Smith, K. L., and R. K. Scott, 2016: The role of planetary waves in the tropospheric jet response to stratospheric cooling. Geophys. Res. Lett., 43, 2904-2911, https://doi.org/10.1002/ 2016GL067849.

Song, Y., and W. A. Robinson, 2004: Dynamical mechanisms for stratospheric influences on the troposphere. J. Atmos. Sci., 61 , 1711-1725, https://doi.org/10.1175/1520-0469(2004)061<1711: DMFSIO $>2.0 . \mathrm{CO} ; 2$.

Tripathi, O. P., and Coauthors, 2015: The predictability of the extratropical stratosphere on monthly time-scales and its impact on the skill of tropospheric forecasts. Quart. J. Roy. Meteor. Soc., 141, 987-1003, https://doi.org/10.1002/qj.2432.

Vitart, F., and Coauthors, 2017: The Subseasonal-to-Seasonal (S2S) Prediction Project database. Bull. Amer. Meteor. Soc., 98, 163-173, https://doi.org/10.1175/BAMS-D-16-0017.1. 